\title{
MORSE/STORM: A \\ GENERALIZED ALBEDO OPTION FOR MONTE \\ CARLO CALCULATIONS
}

I. C. Gomes

P. N. Stevens 
This report has been reproduced directly from the best available copy.

Available to DOE and DOE contractors from the Office of Scientific and Technical Information, P.O. Box 62, Oak Ridge, TN 37831; prices available from (615) 576-8401, FTS 626-8401.

Available to the public from the National Technical Information Service, U.S Department of Commerce, 5285 Port Royal Rd., Springfield, VA 22161.

This report was prepared as an account of work sponsored by an agency of the United States Government. Neither the United States Government nor any agency thereof, nor any of their employees, makes any warranty, express or implied, or assumes any legal liability or responsibility for the accuracy, completeness, or usefulness of any information, apparatus, product, or process disclosed, or represents that its use would not infringe privately owned rights. Reference herein to any specific commercial product, process, or service by trade name, trademark, manufacturer, or otherwise, does not necessarily constiture or imply its endorsement, recommendation, or favoring by the United States Government or any agency thereof. The views and opinions of authors expressed herein do not necessarily state or reflect those of the United States Government or any agency thereof. 
Fusion Energy Division

\title{
MORSE/STORM: A GENERALIZED ALBEDO OPTION FOR MONTE CARLO CALCULATIONS
}

\author{
I. C. Gomes \\ P. N. Stevens
}

The University of Tennessee, Knoxville

Date Published: September 1991

\footnotetext{
Prepared for the Office of Fusion Energy Budget Activity No. AT 15

Prepared by the

OAK RIDGE NATIONAL LABORATORY

Oak Ridge, Tennessee 37831-6285 managed by

MARTIN MARIETTA ENERGY SYSTEMS, INC.

for the

U.S. DEPARTMENT OF ENERGY

under contract DE-AC05-84OR21400
} 


\section{CONTENTS}

LIST OF FIGURES . . . . . . . . . . . . . . . . . . . . . . . . . iv

LIST OF TABLES . . . . . . . . . . . . . . . . . . . . . . . . . v v

ABSTRACT . . . . . . . . . . . . . . . . . . . . . . . v vii

1. INTRODUCTION . . . . . . . . . . . . . . . . . . . . . . . . 1

2. ALBEDOS . . . . . . . . . . . . . . . . . . . . . . . . . . . 2

3. ALBEDO DATA AND SAMPLING PROCEDURES . . . . . . . . . 5

4. MODIFICATION OF THE MORSE/BREESE PACKAGE . . . . . . 7

4.1 THE PSEUDO-PARTICL̃ MODIFICATION . . . . . . . . . . 9

4.1.1 Generation of Pseudo-Particles . . . . . . . . . . . . . . 9

4.1.2 Fair-Game Transport of the Pseudo-Particles . . . . . . . . 11

4.2 INCORPORATION OF A DISPLACED POINT

OF EMERGENCE . . . . . . . . . . . . . . . . . . . . . 12

4.2.1 Determining the Point of Emergence from an
Albedo Event . . . . . . . . . . . . . . . 12

4.2.2 Implementing the Point of Emergence in the

Random Walk . . . . . . . . . . . . . . . . . . . . . 16

4.3 INCORPORATION OF SPATIALLY DEPENDENT

ALBEDO DATA . . . . . . . . . . . . . . . . . . . 16

4.3.1 Generating a New Set of Albedo Data with TORT . . . . . 16

4.3.2 Processing the Spatially Dependent Albedo Data . . . . . . 17

4.3.3 Generation of Adjoint Albedo Data . . . . . . . . . . . . 20

4.3.3.1 Reciprocity relationship for forward and adjunction albedos . . . . . . . . . . . . . . . . . 20

4.3.3.2 Generation procedure and data manipulation . . . . 24

4.3.4 Normalization Factors for Adjoint Calculations . . . . . . . 25

5. RESULTS . . . . . . . . . . . . . . . . . . . . . . . . 28

5.1 THE STANDARD MORSE/BREESE PACKAGE . . . . . . . . 28

5.2 THE PSEUDO-PARTICLE MODIFICATION . . . . . . . . . . 31

5.3 AVERAGE DISPLACEMENT OF THE POINT OF

EMERGENCE .. . . . . . . . . . . . . . . . . . . . . . 35

5.4 GENERATION OF NEW ALBEDO DATA . . . . . . . . . . . 39

5.5 SPATIALLY DEPENDENT ALBEDOS . . . . . . . . . . . . 47

5.5.1 Forward Mode . . . . . . . . . . . . . . . . . . . . . 47

5.5.2 Adjoint Mode . . . . . . . . . . . . . . . . . . . . . 49

6. CONCLUSIONS AND FUTURE WORK . . . . . . . . . . . . . 50

REFERENCES . . . . . . . . . . . . . . . . . . . . . . . . 53 


\section{FIGURES}

2.1. A few of the possible tracks that a particle may experience inside a material medium . . . . . . . . . . . . . . . . . . . . 3

2.2. Albedo reflection process and related phase-space coordinates . . . . 4

4.1. Graphic comparison of (a) full transport, (b) albedo transport, and (c) pseudo-particle/albedo transport modes . . . . . . . . . . . . 8

4.2. Schematic representation of the surface bins used in the MCNP calculations performed to obtain a spatial description of the albedo reflection process for concrete . . . . . . . . . . . . 13

4.3. Displacement in the number of mean free paths as a function of the incident angle for different incident energies in ordinary concrete

4.4. Displacement in the number of mean free paths as a function of the incident energy for different incident angles in ordinary concrete

4.5. Schematic representation of the reflection process using point and spatially dependent albedo data . . . . . . . . . . . . . . . . . 21

4.6. Forward and adjoint azimuthal albedo distribution, for fixed incident energy and angle, and fixed outgoing energy and polar angle . . . . 26

5.1. Geometric configuration of the sample problem . . . . . . . . . . 29

5.2. Shield configuration and detector locations for the sample problem . . 30

5.3. Angular position of the emergent particles in relation to the point of incidence . . . . . . . . . . . . . . . . . . . . . . . . . 35

5.4. Radial distance from the point of incidence to the point of emergence . . . . . . . . . . . . . . . . . . . 36

5.5. Angular azimuthal distribution of the available albedo data and of the MCNP calculated data . . . . . . . . . . . . . . . . . . . 42

5.6. Angular azimuthal distribution of the CARP albedo data and of the MCNP albedo information . . . . . . . . . . . . . . . . . . 42

5.7. Angular distributions of various albedo data sets . . . . . . . . . . . 44

5.8. Spatial distributions of the TORT albedos . . . . . . . . . . . . 46 


\section{TABLES}

5.1. Results for benchmark cases (full-transport MORSE and MCNP) and for the standard MORSE/BREESE package using next-event and boundary-crossing estimators . . . . . . . . . . . . . . . . .

5.2. Results for benchmark case (full-transport MORSE) and for the MORSE/BREESE package using next-event and boundary-crossing estimators with full transport at the corners . . . . . . . . . . .

5.3. Results using pseudo-particles without displacement of the point of emergence . . . . . . . . . . . . . . . . . . . . . .

5.4. Results using pseudo-particles without displacement of the point of emergence and with full transport at the corners

5.5. Results using pseudo-particles with displacement of the point of emergence in the direction of the incident particle projected on the albedo plane . . . . . . . . . . . . . . . . . . . . .

5.6. Results using pseudo-particles with displacement of the point of emergence in the direction of the incident particle projected on the albedo plane and full transport at the corners

5.7. Results using pseudo-particles with displacement of the point of emergence in the direction of the axis of the duct . . . . . . . .

5.8. Results using pseudo-particles with displacement of the point of emergence in the direction of the axis of the duct and full transport at the corners . . . . . . . . . . . . . . . . . . . .

5.9. Limits of the polar angles of the CARP and MCNP albedo data and of the polar angles of the TORT albedo data . . . . . . . . . . .

5.10. Limits of the azimuthal angles of the CARP and MCNP albedo data and of the TORT albedo data

5.11. MORSE/STORM results with spatially dependent albedos and MORSE benchmark results . . . . . . . . . . . . . . . . . . . 48

5.12. Results of adjoint calculations using the MORSE/STORM code package for the sample problem

5.13. Adjuncton-albedo calculation and MORSE and MCNP benchmark results for the sample problem 


\begin{abstract}
The advisability of using the albedo procedure for the Monte Carlo solution of deep penetration shielding problems that have ducts and other penetrations has been investigated. The use of albedo data can dramatically improve the computational efficiency of certain Monte Carlo calculations. However, the accuracy of these results may be unacceptable because of lost information during the albedo event and serious errors in the available differential albedo data. This study was done to evaluate and appropriately modify the MORSE/BREESE package, to develop new methods for generating the required albedo data, and to extend the adjoint capability to the albedo-modified calculations. Major modifications to MORSE/BREESE include an option to save for further use information that would be lost at the albedo event, an option to displace the point of emergence during an albedo event, and an option to use spatially dependent albedo data for both forward and adjoint calculations, which includes the point of emergence as a new random variable to be selected during an albedo event. The theoretical basis for using TORT-generated forward albedo information to produce adjuncton albedos was derived. The MORSE/STORM package was developed to perform both forward and adjoint modes of analysis using spatially dependent albedo data. Results obtained with MORSE/STORM for both forward and adjoint modes were compared with benchmark solutions. Excellent agreement and improved coniputational efficiency were achieved, demonstrating the full utilization of the albedo option in the MORSE code.
\end{abstract}




\section{INTRODUCTION}

The albedo for a reflecting medium is defined as the ratio between the emergent current and the incident current. The solution of radiation transport problems can be simplified by allowing part of the geometry to be represented by albedo surfaces. Significant savings in computer time can be achieved, as well as a better description of the radiation field within specific regions of interest.

Monte Carlo calculations can benefit from the albedo concept, since the computation time can be reduced by not tracking particles inside albedo regions, such as the walls of ducts and cavities. However, this advantage is not realized without some cost; the generation of albedo data is very expensive, and the amount of data can be prohibitively large.

An albedo procedure is available as an option the MORSE Monte Carlo code. ${ }^{1}$ The multigroup energy format of the MORSE code facilitates the coupling of albedo information generated by discrete ordinates with the Monte Carlo code.

On the basis of early applications of the albedo procedure to Monte Carlo analyses, it was recommended that albedos should be used only for problems in which the characteristic dimensions of the penetrations are large when compared with the radiation particle's mean free path in the albedo media. To better understand the reasons behind this restriction and to develop a basis for overcoming this problem, a set of benchmark-quality, full-transport calculations was performed using MORSE and the MCNP $\operatorname{code}^{2}$ for a wide range of problems. The results were compared with results from Monte Carlo/albedo calculations performed with the MORSE/BREESE code package. ${ }^{3}$ The albedo-driven results, despite achieving solutions with essentially zero standard deviations and requiring reasonable amounts of computer time, performed very poorly in terms of accuracy; they consistently underestimated the benchmark results, in some cases by orders of magnitude.

As a first step toward generalizing the albedo procedure, the standard MORSE/BREESE package ${ }^{3}$ was modified to recover some of the information lost at the albedo events. The results of calculations with the modified version of the code were much more accurate, $l$ at they were consistently about $20 \%$ lower than the benchmark results. A plausible explanation for this deviation is that the albedo data did not correctly represent the actual reflection process.

A series of MCNP calculations was performed to benchmark the standard albedo data. The Monte Carlo results and the standard albedo azimuthal distributions were found to be significantly different, probably because the angular flux files from the discrete ordinates code $\mathrm{DOT}^{4}$ contained negative angular fluxes for a few combinations of energy groups and directions of the quadrature set.

The three-dimensional (3-D) discrete ordinates code TORT $^{5}$ was modified and used to generate a new set of albedo data that inclucles a spatial variable for the point of emergence. The characteristic method for the flux sweep was used to ensure positive angular fluxes for all energy groups and discrete directions. An experimental package of codes called MORSE/STORM was developed to format 
maniuplate, and use the generated albedo data. When spatially dependent albedo data from the modified version of the TORT code are used, the subroutines and interface codes that make up the BREESE system of the MORSE/BREESE package are replaced by the STORM system routines.

The new procedures described in this report significantly broaden the applicability of albedo procedures. Improvements in the figure of merit should be possible through the use of appropriate biasing techniques, which was not formally investigated in this research.

Section 2 presents the fundamentals of the albedo concept, along with descriptive definitions of various kinds of albedos. In Sect. 3 , the available albedo data are described and sampling procedures are discussed. Section 4 describes the modifications introduced into the MORSE/BREESE package, the new method for calculating spatially dependent albedo data, the developinent of the MORSE/STORM package, the derivation of the reciprocity relationship between forward and adjoint albedos, and the implementation of the adjoint capability in the MORSE/STORM package. In Sect. 5, calculations and results are presented. Finally, the conclusions of this research and recommendations for future work are presented in Sect. 6.

\section{ALBEDOS}

Tnlike the reflection of the light, which is mainly a surface phenomenon, neutrons and gamma rays are scattered back from inside the material-sometimes from depths of several mean free paths and after several collisions. Figure 2.1 shows a few of the tracks that a particle may experience inside a material medium. For neutrons, the scattering from nuclides can be elastic or inelastic, and Compton scattering from electrons is dominant for photons. As a result, a particle can emerge through the same surface that it entered. This can be described in the macroscopic sense by a reflection coefficient that is usually called the albedo. The albedo for a reflecting medium is defined as the ratio between the emergent current and the incident current,

$$
\alpha\left(E_{0}, \Theta_{0} ; E, \Theta, \phi\right)=\frac{J(E, \Theta, \phi)}{J\left(E_{0}, \Theta_{0}\right)},
$$

where $J\left(E_{0}, \Theta_{0}\right)$ is the incident current, $J(E, \Theta, \phi)$ is the emergent current, and $\alpha\left(E_{0}, \Theta_{0} ; E, \Theta, \phi\right)$ is the albedo, as shown in Fig. 2.2.

If the spatial dependence of the point of emergence is included, the equation for the albedo becomes

$$
\alpha\left(E_{0}, \mathbf{r}_{0}, \Theta_{0} ; E, \mathbf{r}, \Theta, \phi\right)=\frac{J(E, \mathbf{r}, \Theta, \phi)}{J\left(E_{0}, \mathbf{r}_{0}, \Theta_{0}\right)}
$$

where $\mathbf{r}$ represents the point of emergence and $\mathbf{r}_{0}$ represents the point of incidence.

Albedos are available in several forms. The for $n$ used in Eq. (2.1) is doubly differential; that is, it is differential with respect to both the reflected energy $E$ and 


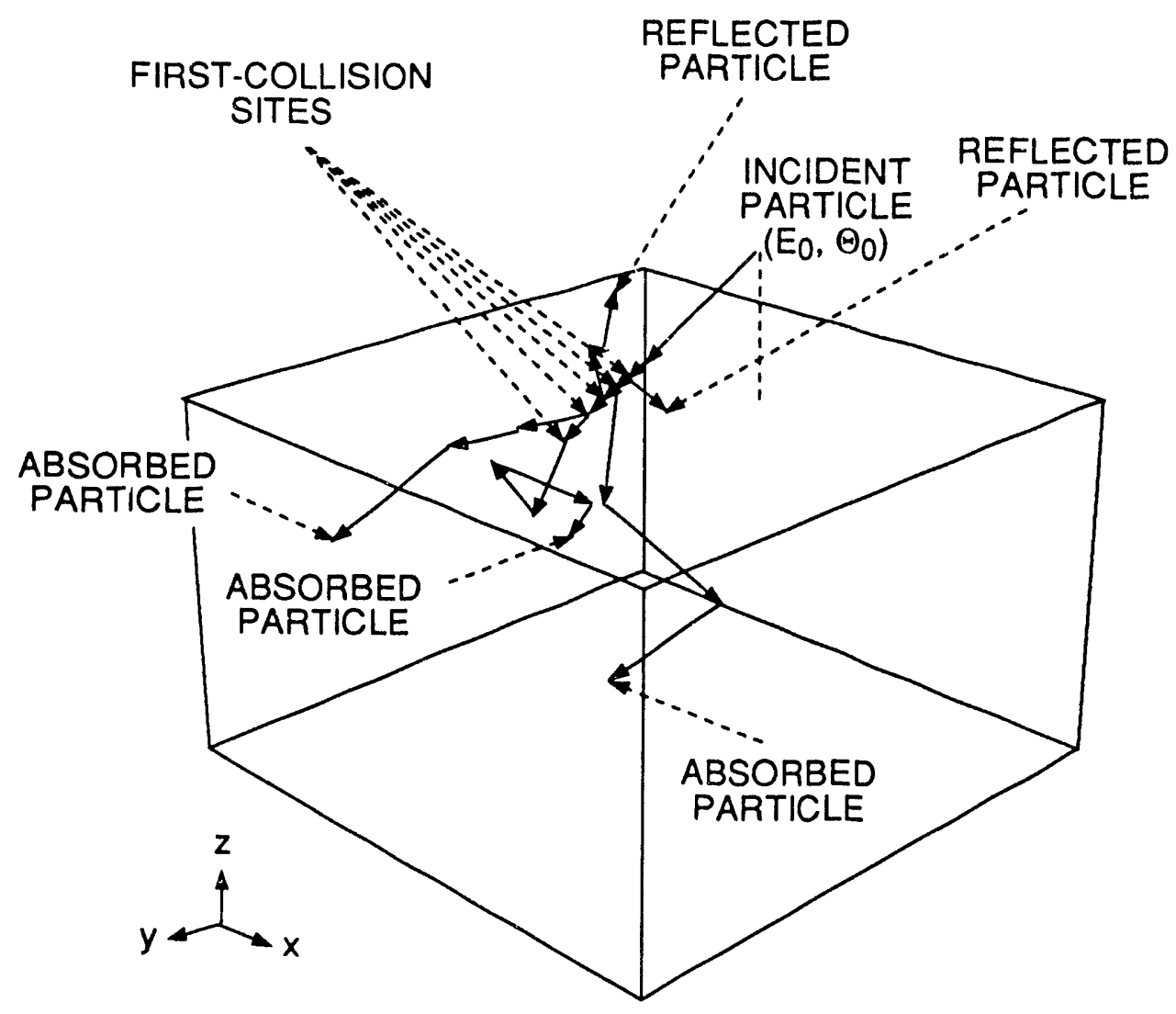

Fig. 2.1. A few of the possible tracks that a particle may experience inside a material medium.

the reflected direction (described by $\Theta$ and $\phi$ ). Equation (2.2) represents a triply differential albedo, which includes the dependence on the spatial point of emergence. The doubly differential albedo may be obrained from the triply differential albedo through integration over the spatial variable. A singly differential albedo is obtained when a doubly differential albedo is integrated over either energy or direction.

The portion of the surface through which backscattered particles leave a reflecting medium can be called the reflecting region. If a beam of particles is incident on the surface of a reflecting medium, the reflecting region of this surface is always larger than the intersection of the beam with the surface. If the dimensions of the reflecting region are considerably smaller than the distance from the reflecting region to the detector, the dimensions of the reflecting region can be neglected and the reflecting region may be considered coincident with the intersection of the incident beam with the surface. In such cases, the point of emergence can be considered coincident with the point of incidence, and a knowledge of the doubly 


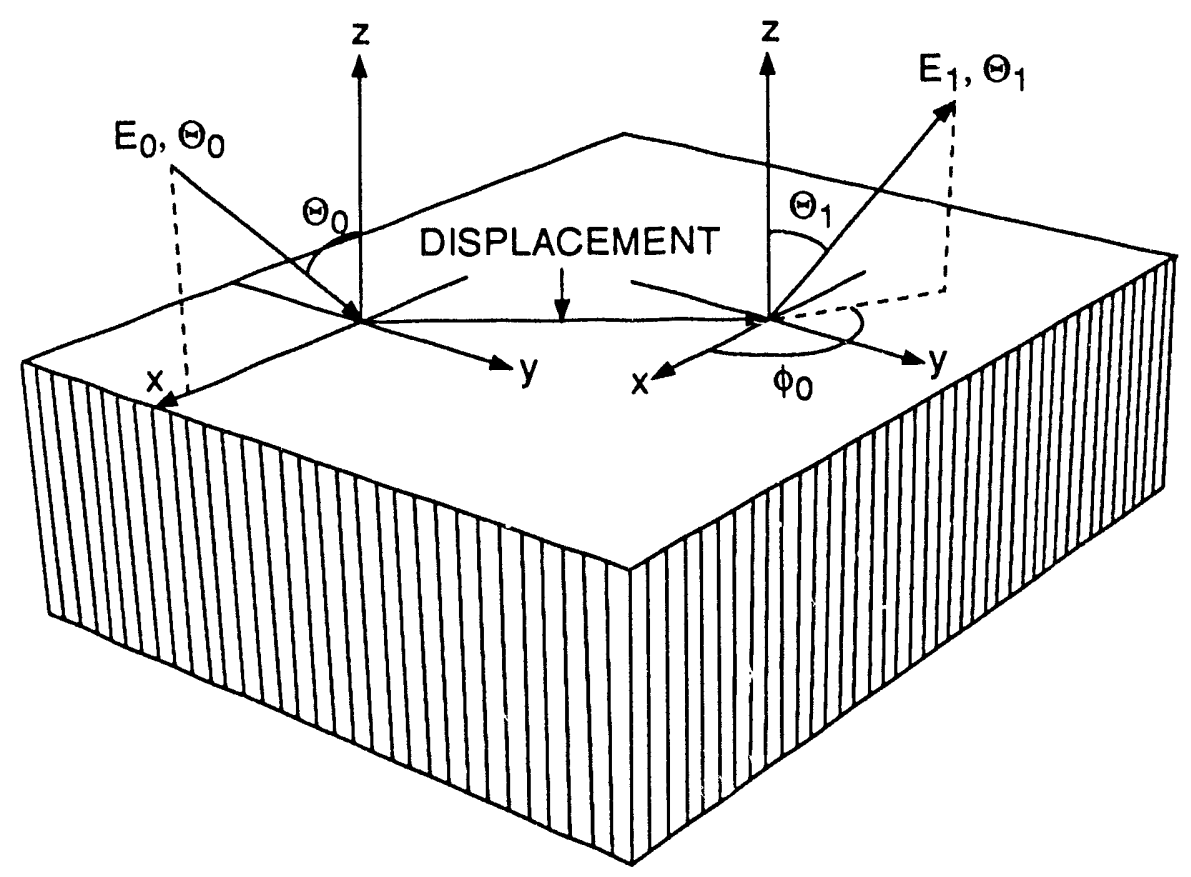

Fig. 2.2. Albedo reflection process and related phase-space coordinates.

differential albedo is sufficient. The available albedo data are, in fact, "point" albedos. Point albedos are doubly differential and should be used only for problems in which the distance to the detector is large compared with the dimensions of the reflecting region. This restricts the application of doubly differential albedos to regions far from the detectors or, in the case of ducts and cavities, to problems for which the diameter of the duct is large enough to conply with this constraint.

Other representations of albedo data are the differential number albedo,

$$
\alpha_{N t}\left(E_{0}, \Theta_{0} ; \Theta, \phi\right)=\int_{E=0}^{E=E_{0}} \alpha\left(E_{0}, \Theta_{0}, E^{\prime}, \Theta, \phi\right) d E^{\prime}
$$

the polar differential number albedo,

$$
\alpha_{N a}\left(E_{0}, \Theta_{\wp}, \Theta\right)=\int_{\phi=0}^{\phi=2 \pi} \alpha_{N t}\left(E_{0}, \Theta_{0}, \Theta, \phi\right) d \phi,
$$

and the total number albedo,

$$
\alpha\left(E_{0}, \Theta_{0}\right)=\int_{\mu=0}^{\mu=1} \alpha_{N a}\left(E_{0}, \Theta_{0}, \Theta\right) d \mu
$$

where $\mu=\cos \Theta$. 
Using these albedos, which by their nature are less descriptive, can speed up the sampling process in a Monte Carlo calculation if the variables are selected using marginal and conditional probability distribution tables. This point is discussed in more detail in Sect. 4.

\section{ALBEDO DATA AND SAMPLING PROCEDURES}

Empirical or analytical formilas for albedo data would be very convenient for Monte Carlo calculations, but because of the detail and resolution required for accurate solutions of complex problems, the available albedo formulations are not suitable. Differential albedo information is most commonly obtained through twodimensional (2-D) discrete ordinates calculations. The usual problem consists of a reflecting semi-infinite medium with an incident monodirectional beam of particles having energies within a particular energy group. A run is performed for each combination of discrete incident polar direction and energy group. The angular and energy distributions of the reflected particles are calculated, and these distributions are expressed as a reflected current per unit incident current. A Monte Carlo code such as MORSE can use this albedo information to reflect particles that reach an albedo surface. The phase-space coordinates of the reflected particle are selected according to probability distribution tables, which are constructed from the angular flux output of the discrete ordinates code. These point albedo data provide no information about the spatial distribution of the emergent particles.

The MORSE/BREESE package was developed to use albedo information as a means of simplifying the Monte Carlo generation of random walks. The BREESE module comprises the subroutines ALBIN, ALBDO, ALBDOE, and THETO and a separate program that writes a tape of albedo data for input into MORSE. The subroutine ALBIN reads the albedo tape and sets the pointers for the common block. The subroutine ALBDO performs the reflection process, sampling from the probability tables for a given incident polar angle (as defineci by the subroutine THETO), outgoing energy group, and direction. The subroutine ALBDOE estimates the statistically defined next-event contribution to the detectors from each albedo event. Cumulative distribution functions (cdf's) are constructed for each of the statistically dependent variables (outgoing polar angle, azimuthal angle, and energy) to avoid the excessive amount of computer time that would be spent searching a single joint cdf.

The total albedo $a\left(E_{0}, \Theta_{0}\right)$ may be described as the probability that a particle will be reflected from the reflecting medium (independent of the outgoing energy and angle). The $\alpha\left(E_{0}, \Theta_{0}\right)$ is used to adjust the weight of a particle that undergoes an albedo event. The weight of the incident particle is multiplied by the total albedo. This correction of the weight of the particle is statistical in nature and represents the average number of particles emerging from a reflecting medium following an 
albedo event that involves an incident particle with a particular energy and direction $\left(E_{0}, \Theta_{0}\right)$.

To select the outgoing phase-space coordinates (group $g, \Theta, \phi$ ), the following cdf's are constructed. The marginal cdf for the outgoing polar angle is given by

$$
\operatorname{cdf}_{\Theta}\left(E_{0}, \Theta_{0} ; \Theta\right)=\frac{\int_{\mu^{\prime}=0}^{|\mu|} d\left|\mu^{\prime}\right| \alpha_{N a}\left(E_{0}, \Theta_{0}, \Theta\right)}{\alpha\left(E_{0}, \Theta_{0}\right)},
$$

where $\mu=\cos \Theta$ and $\alpha_{N a}\left(E_{0}, \Theta_{0}, \Theta\right)$ is the polar differential number albedo defined in Eq. (2.5).

The conditional cdf for the azimuthal angle within the polar angle sampled from the previous cdf is

$$
\operatorname{cdf}_{\phi}\left(E_{0}, \Theta_{0}, \Theta ; \phi\right)=\frac{\int_{0}^{\phi} d \phi^{\prime} \alpha_{N t}\left(E_{0}, \Theta_{0} ; \Theta, \phi\right)}{\alpha_{N a}\left(E_{0}, \Theta_{0}, \Theta\right)},
$$

where $\alpha_{N t}\left(E_{0}, \Theta_{0} ; \Theta, \phi\right)$ is the differential number albedo defined in Eq. (2.4).

The doubly conditional cdf for the energy for the previously defined outgoing direction is

$$
\operatorname{cdf}_{E}\left(E_{0}, \Theta_{0}, \Theta, \phi ; E\right)=\frac{\int_{E^{\prime}=0}^{E} d E^{\prime} \alpha\left(E_{0}, \Theta_{0} ; E, \Theta, \phi\right)}{\alpha_{N t}\left(E_{0}, \Theta_{0} ; \Theta, \phi\right)},
$$

where $\alpha\left(E_{0}, \Theta_{0} ; E, \Theta, \phi\right)$ is the doubly conditional differential albedo.

These definitions for the cdf's are for continuous variables. For albedos generated with discrete ordinates codes, the energy and angular variables are discretized. Thus, values associated with discrete segments of those variables are sampled instead.

In the MORSE/BREESE package, the subroutine ALBIN reads the cdf's and the subroutine ALBDO selects the outgoing phase-space coordinates. First, the subroutine THETO calculates the cosine of the incident polar angle $\Theta$,

$$
\cos \Theta=u_{n} u+v_{n} v+w_{n} w
$$

where $u_{n}, v_{n}$, and $w_{n}$ define the cosines of the normal to the surface, and $u, v$, ard $w$ are the direction cosines of the incoming particle.

The incident polar angle $\Theta$ is compared with the limits of each incoming angular polar bin of the albedo data, and the bin to which $\Theta$ belongs is identified. The discrete form of $\mathrm{cdf}_{\Theta}[\mathrm{Eq} .(3.1)]$ is used to select an outgoing polar angle bin. The outgoing polar angle is then selected from an equally probable distribution within the limits of the selected polar angle bin. The outgoing azimuthal angle $\phi$ bin is selected from the conditional cumulative probability distribution $\operatorname{cdf}_{\phi}[\mathrm{Eq}$. (3.2)]. Like the polar angle, the outgoing azimuthal angle is selected from an equally probable distribution within the limits of the selected azimuthal angle bin. Finally, 
the outgoing energy bin is selected from the doubly conditional $\operatorname{cdf}_{E}$ [Eq. (3.3)]. If the group structure for the cross sections is different from that of the albedo data, the outgoing energy group is sampled from an equally probable distribution over lethargy.

In the sampling scheme implemented in the BREESE code, energy cutoff cannot be used because the angular distribution from which the sampling process is initiated is a summation over all possible outcomes, and a fair game is not preserved when using energy cutoff unless the angular distributions for all outgoing energy groups are exactly the same. Albedo data are usually generated for coupled (neutrongamma ray) problems, so using an angular distribution that is associated with the summation over all energy groups as the starting point for the sampling process limits the applicability of energy cutoff as well as that of energy biasing during the random walk. Angular biasing is still a fair game with the implemented sampling scheme.

Analysis of an albedo data set distributed by the Radiation Shielding Information Center (RSIC) at Oak Ridge National Laboratory revealed negative angular fluxes in the basic data. These negative angular fluxes cannot be avoided because of the low-order expansion of the scattering cross sections. They occur mainly in the high-energy groups for the forward component of the outgoing angular flux. This leads to an undesirable distortion in the description of the reflection process and to an underestimate of the forward component, which is usually the most important component of the radiation field being analyzed. The BREESE routines treat these negative fluxes as if they were a part of the true solution. Since the negative fluxes lead to negative probabilities, the sign of the particle's statistical weight is reversed in ranges where the probability density function is negative.

\section{MODIFICATION OF THE MORSE/BREESE PACKAGE}

The original MORSE/BREESE package was designed to perform the computational steps needed to accomplish the albedo-modified random walk. Particles are followed, or tracked, only within zones enclosed by the albedo surfaces. The computational time is minimized because the tracking (ray tracing) is performed only between albedo events, and much of the random walk that would have occurred within the albedo medium is in effect accomplished by the deterministic reflection of the particle. Problems in which streaning through ducts or passageways is the dominant component of the radiation field can be solved very efficiently using the albedo option.

The MORSE/BREESE code package can handle forward calculations that combine albedo reflection and full transport for selected regions. Effects of interest (responses of detectors that view an albedo surface) can be estimated by using either next-event estimation or an analog boundary-crossing estimator, as discussed in Sect. 5. For albedo reflection, a particle suffers a collision-like event when it 
encounters an albedo suface. The weight of the incident particle is multiplied by the total albedo, and the outgoing energy and direction are selected according to the joint probability distribution associated with the doubly differential albedo.

Because the albedo-modified random walk procedure used by the standard MORSE/BREESE code package does not ailow particle transport inside albedo regions, the radiation field at positions behind albedo surfaces cannot be estimated. To correct this situation, the MORSE code was modified to include pseudoparticles, which permit statistical estimates at positions behind albedo surfaces. The three possible transport modes (full, albedo, and pseudo-particle) are compared in Fig. 4.1. The pseudo-particle modification increases the computational time but

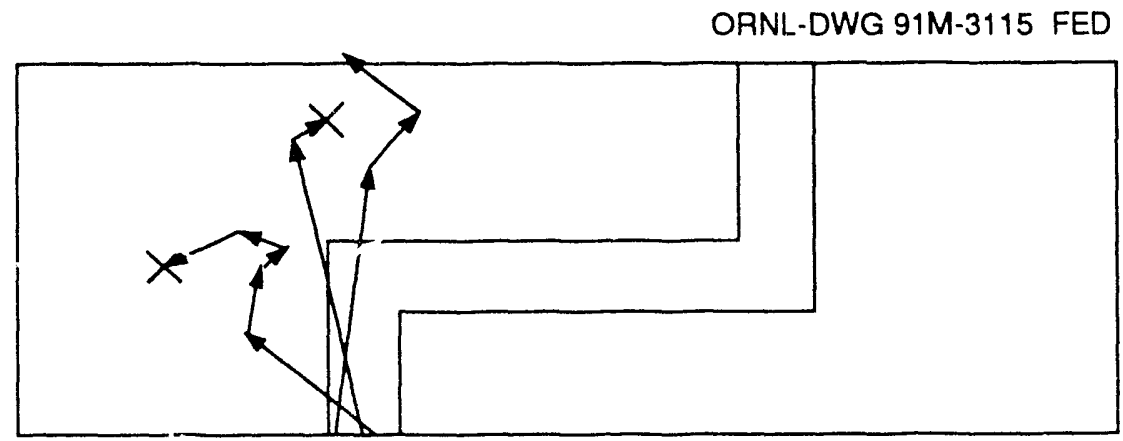

(a) FULL TRANSFORT

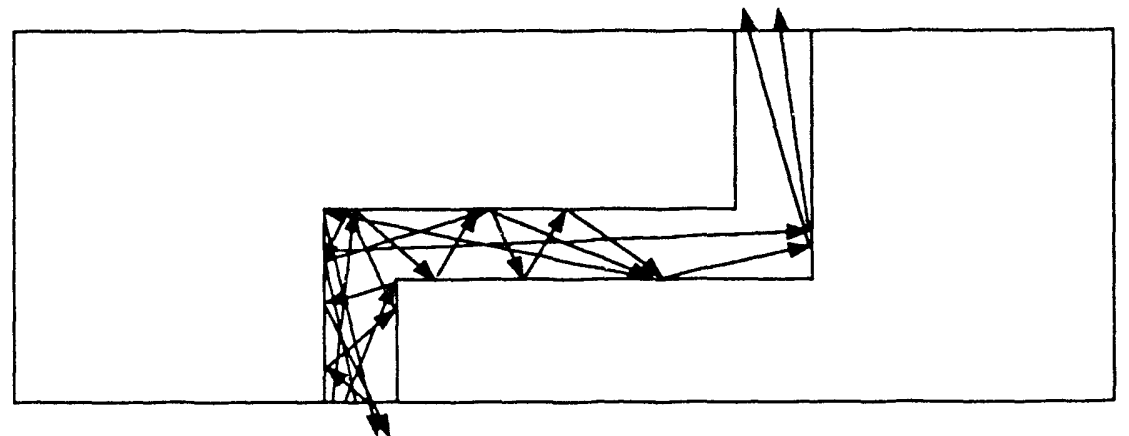

(b) ALBEDO TRANSPORT

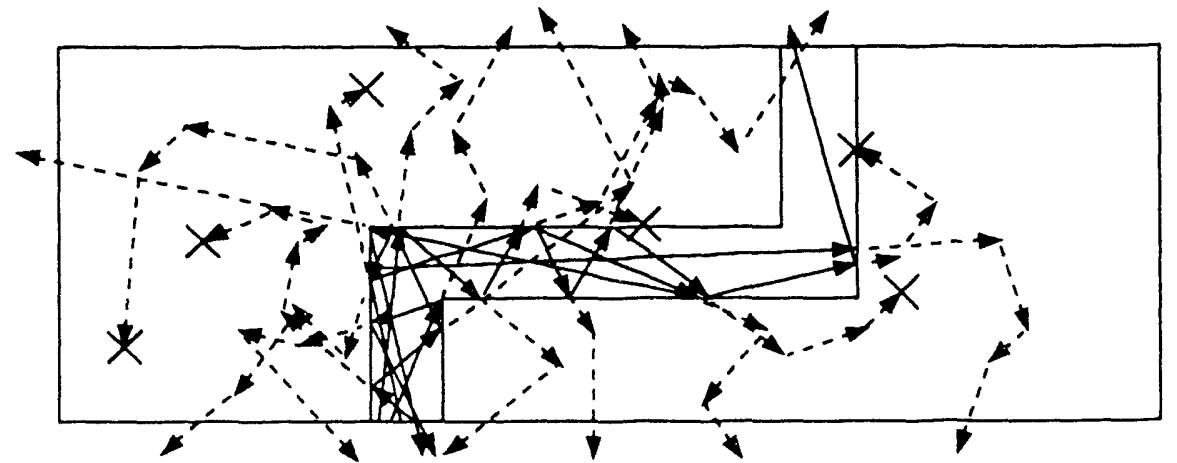

(c) PSEUDO-PARTICLE/ALBEDO TRANSPORT

Fig. 4.1. Graphic comparison of (a) full transport, (b) albedo transport, and (c) pseudo-particle/albedo transport modes. 
provides more precise results and a more complete description of the radiation field for use in statistical estimates. The amount of computer time required can be much smaller than for the full-transport case because of the advantage gained when particles are driven deterministically by the albedo reflections through the duct, with pseudo-particles being created at positions not easily reached by normal transport.

\subsection{THE PSEUDO-PARTICLE MODIFICATION}

In the standard version of the MORSE/BREESE package, collisions that could occur inside the albedo medium and statistically contribute to effects of interest outside the albedo surface envelope cannot be represented by the reflected portion of the particle. "To overcome this problem, the "pseudo-particle" modification was introduced into the MORSE code to allow the transport of particles inside an albedo medium and the statistical estimation of effects of interest outside the albedo surface envelope. The albedo reflection process is kept unmodified, and a pseudoparticle may be created at each albedo event, depending on the survival probability associated with that particular albedo event. As soon as a pseudo-particle is created, it is stored in the neutron bank with the same direction, position, and energy as the incident albedo-transported particle, and the tracking of the albedo reflected particle is resumed. The pseudo-particles are tracked after the source particles that make up a given batch are completely processed. The Monte Carlo transport of a pseudo-particle is performed as it would be in a full-transport calculation, allowing the use of any of the available variance-reduction biasing techniques.

\subsubsection{Generation of Pseudo-Particles}

The new procedure was implemented by specifying a second set of medium numbers for the albedo media, which is used when following the pseudo-particles within the albedo medium. The user may control the generation of pseudo-particles through mechanisms that inclucle the accumulated average weight of the albedotransported particles, the importance by direction, or the specification of regionand energy-dependent probabilities for the generation of pseudo-particles.

The default procedure is the comparison of the weight of an albedo-transported particle with the accumulated arcrage weight of the albedo-transported particles by region and energy. The weight of an albedo-transported particle that suffers an albedo event is compared with the average weight of all particles that have previously experienced albedo events within that portion of phase space. This procedure controls the number of particles generated by preventing large fluctuations in the weights of the generated pseuclo-particles. An initial guess of the average weight is prorided at the start of a run by processing a dummy batch of a user-specified number of particles. During the albedo-modified Monte Carlo analysis, the average weight of the albedo-transported particles is updated after 
each batch. The average weight by region and by energy group is used in a fairgame procedure to determine whether an albedo collision will produce a pseudoparticle. At each albedo event, the current weight of the albedo-transported particle is compared with the "average weight" associated with the region and energy group of the incident particle. If the weight of the albedo-transported particle is less than the average weight, a Russian roulette game is played. The probability for survival is

$$
P_{\mathrm{s}}=\frac{\text { WGTINC }}{\text { WGTAVG }\left(E_{\mathrm{in}}\right)},
$$

where WGTINC is the weight of the incident albedo-transported particle and WGTAVG $\left(E_{\mathrm{in}}\right)$ is the batch estimate of the appropriate "average weight." If a random number is less than or equal to $P_{s}$, a pseudo-particle is banked with a weight equal to WGTAVG $\left(E_{\mathrm{in}}\right)$. If the weight of a pseudo-particle is larger than the average weight, the pseudo-particle is banked with its current weight.

The importance-by-direction procedure for generating pseudo-particles is based on the angle $\Theta_{D}$ between the direction of the incoming albedo-transported particle and the vector connecting the albedo-event point to a "point of interest." The biasing is accomplished in terms of $\left[\cos \left(\Theta_{\mathrm{D}} / 2\right)\right]^{n}$, where $n$ is an integer provided by the user; when $n=0$, the biasing procedure is turned off. The code determines $\Theta_{D}$ from the formula

$$
\overline{\mathbf{n}} \cdot \overline{\mathbf{a}}=\|\overline{\mathbf{n}}\| \cdot\|\overline{\mathbf{a}}\| \cos \left(\Theta_{\mathrm{D}}\right),
$$

where $\overline{\mathbf{n}}$ is the inward normal to the plane where the albedo event occurs and $\overline{\mathbf{a}}$ is the vector connecting the point of the albedo event to the point of interest. Equation (4.2) can be rewritten as

$$
\cos \Theta_{D}=\frac{u\left(x_{\mathrm{imp}}-x\right)+v\left(y_{\mathrm{imp}}-y\right)+w\left(z_{\mathrm{imp}}-z\right)}{\text { dist }}
$$

where

$$
\text { dist }=\left[\left(x_{\mathrm{imp}}-x\right)^{2}+\left(y_{\mathrm{imp}}-y\right)^{2}+\left(\tilde{z}_{\mathrm{imp}}-z\right)^{2}\right]^{1 / 2}
$$

$u, v$, and $w$ are the direction cosines of the albedo particle; $x, y$, and $z$ are the coordinates of the albeclo event; and $x_{\mathrm{imp}}, y_{\mathrm{imp}}$, and $z_{\mathrm{inp}}$ are the coordinates of the point of interest.

The survival probability is based on the half-angle $\Theta_{D} / 2$. This ensures that $\cos \Theta_{D} / 2$ will assume a value between zero and one. A Russian roulette game is played by comparing a random number with $\left[\cos \left(\Theta_{\mathrm{D}} / 2\right)\right]^{n}$. If the random number is greater than $\left[\cos \left(\Theta_{\mathrm{D}} / 2\right)\right]^{n}$, no pseuclo-particle is generated. If a pseudo-particle is generated, it is stored in the bank with its weight corrected by the factor $[1 / \cos (\Theta / 2)]^{n}$. To prevent the occurrence of particles with very large or even infinite weights, a weight correction factor of 10 is applied to surviving pseudo-particles if $[\cos (\Theta / 2)]^{n}$ is less than 0.2 .

The modified input file also provides an option for assigning probabilities for generating seudo-particles by region and by energy group. The generation 
probability associated with the incident albedo-transported particle is compared to a random number. If the random number is smaller than the generation probability, a pseudo-particle is generated with the weight of the albedo-transported particle multiplied by the inverse of the probability.

Any of these biasing procedures can be turned off by the user. Because these procedures may generate pseudo-particles with very large weights, the user must exercise some caution in the choice of biasing parameters.

\subsubsection{Fair-Game Transport of the Pseudo-Particles}

An albedo-transported particle is reflected when it encounters an albedo surface. Its statistical weight is multiplied by the total albedo in order to correctly represent the statistical nature of the reflection process. A pseudo-particle is created with the same phase-space coordinates as the inciclent particle. To preserve the fair game, pseudo-particles are not permitted to cross back through the surface of the albedo medium because an albedo-reflected particle is only one of the possible outcomes from the full transport of a particle within the albedo medium. If a pseudo-particle attempts to cross back through the albedo surface, it is eliminated, or killed, because this event (reflection) is deterministically represented by the albedo data.

To eliminate pseudo-particles that try to cross back through the albedo surface, a check is made at each collision site to determine if the ray connecting two successive collision sites intersects the albedo surface. If an intersection of the albedo surface is detected, the pseudo-particle is killed. When several albedo surfaces are used, the check is performed only with respect to the albedo surface at which the pseudoparticle was created. To accomplish this, two different hemispheres are associated with each albedo surface, and within each hemisphere only one type of particle is allowed to exist. Also, when next-event estimation is used, pseudo-particles are not allowed to contribute to detectors for which the straight line connecting the collision point to the detector intersects the albedo surface at which the pseudo-particle was created.

Albedo data can be generated for media having planar, cylindrical, or spherical surfaces. The relative position of a point to a planar surface is

$$
\mathrm{SIGN}=\overline{\mathbf{n}} \cdot \overline{\mathbf{c}}
$$

where $\overline{\mathbf{n}}$ is the outward normal with respect to the albedo plane and $\overline{\mathbf{c}}$ is the vector connecting the collision point to the point where the pseudo-particle was created. Equation (4.5) can be rewritten as

$$
\operatorname{SIGN}=u_{n}\left(x_{0}-x\right)+v_{n}\left(y_{0}-y\right)+w_{n}\left(z_{0}-z\right)
$$

where $u_{n}, v_{n}$, and $u_{n}$ are the direction cosines of the outward normal to the plane; $x_{0}, y_{0}$, and $z_{0}$ are the coorclinates of a point on the plane; and $x, y$, and $z$ are the coordinates of the point for which the relative position to the plane is clesired. 
A positive value of SIGN indicates that the point $(x, y, z)$ is within the same hemisphere as the positive outward normal of the surface. In applying this equation to the Monte Carlo transport of pseudo-particles, the $(x, y, z)$ are the coordinates of the pseudo-particle's next collision site. As long as the value of SIGN is negative, the tracking of the pseudo-particle continues. If a positive value of SIGN is calculated, the pseudo-particle is killed.

For next-event estimation, essentially the same procedure is used. The coordinates of the detector are used in place of the coordinates of the collision point $(x, y, z)$ in Eq. (4.6). The pseudo-particle will not make a contribution to a detector if SIGN corresponding to that detector is positive.

For cylindrical or spherical geometry, the only check performed is to determine if the distance from the collision point to the axis of the cylinder on the radial plane or to the center of the sphere, respectively, is larger than the defining radius of the body under consideration. Since albedo media are usually placed at the external region of the cylinder or sphere, the pseudo-particle will be killed if it tries to enter the body.

A pseudo-particle is killed if it cannot make contributions to any of the detectors specified in the problem. This follows from Eq. (4.6) because the sign of SIGN changes only if a pseudo-particle crosses back through the albedo surface, which results in a fseudo-particle kill. This procedure saves computing time by preventing the following of pseudo-particles that cannot contribute.

\subsection{INCORPORATION OF A DISPLACED POINT OF EMERGENCE}

\subsubsection{Determining the Point of Emergence from an Albedo Event}

Traditional point albedos are generated by considering the point of emergence from an albedo-type collision to be coincident with the point of incidence. The use of such point albedos is considered acceptable and/or reasonably accurate if the distance traveled by particles between two successive albedo events is large compared with the mean free path of the radiation in the albedo medium. In other words, as stated in Sect. 2, the dimensions of the reflecting region must be small compared with the distance between the albedo event and the detector.

Using point albedos in problems in which the radiation field has streaming components has the effect of retarding the transport of the albedo-transported particles. As a consequence, detector responses downstream are likely to be underestimated. This effect can be expected on phenomenological grounds: because of preferential scattering in the forward direction, the reflected particle has a higher probability of emerging at positions downstream from the point of incidence.

The most common applications of the albedo concept are problems in which particles stream inside ducts and cavities. Point albedo data can be used only 
for configurations in which the internal diameter of the duct (or cavity) is large compared with the radiation particle's mean free path.

Two different approaches are used in this research to mitigate this limitation. The first is the generation of a new set of albedo data with the advantage of correcting the distortions that exist in the available sets of albedo data. This approach is discussed in Sect. 4.3. The second is to infer, from Monte Carlo calculations, appropriate displacement corrections to the available point albedo data. The formulation of an empirical relation to describe the displacement of the emergent particle relative to the point of incidence is discussed here.

The major problem in incorporating this modification into the albedo-modified random walk was that the information needed to calculate the "average displacement" is generally unavailable. New calculations were needed to provide this information.

A set of albedo-type calculations was performed with the MCNP code for various combinations of the angle and energy of the incident source particles. The weights of the reflected particles were counted within spatial bins on the albedo surface. Figure 4.2 shows a schematic representation of the surface bins used in the calculations. Only the total albedo for each surface area bin was estimated.

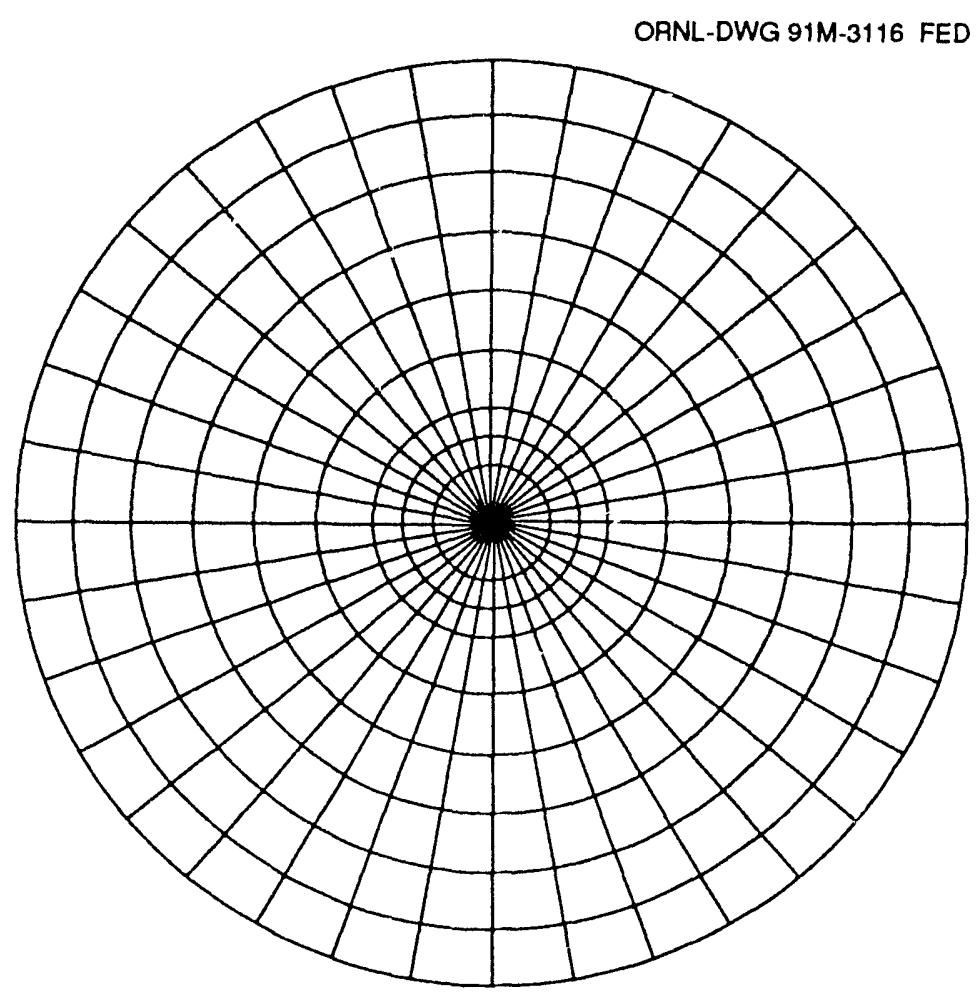

Fig. 4.2. Schematic representation of the surface bins used in the MCNP calculation to obtain a spatial description of the albedo reflection process for concrete. 
The results for each surface bin were projected onto the direction defined by the intersection of the albedo plane with the plane normal to the albedo plane, which contains the direction of the inciclent source particles (the projection was performed as if each bin were represented by a vector with its origin at the point of incidence of the source particles, with a direction defined by the relative position of the bin with respect to the line of projection and a magnitude equal to the distance from the point of incidence multiplied by the total albedo computed at the bin). By summing over all projected vectors, a single distance (in mean free paths) between the point of incidence and the "average point of emergence" was obtained for each incident angle and energy combination. Figure 4.3 displays the average distance (in mean free paths) as a function of the angle of incidence for the energies considered. A new average distance that depends only on the angle of inciclence can be determined. Figure 4.4 displays the incident energy of the particle as a function of the average number of mean free paths from the incident point for each of the incident angles. The average number of mean free paths is not independent of the incident energy of the particle, but the dependence is clearly weaker than the dependence on the angle of incidence. Therefore, the dependence on incident energy was neglected, thus keeping the empirical results and required Monte Carlo procedures as simple as possible.

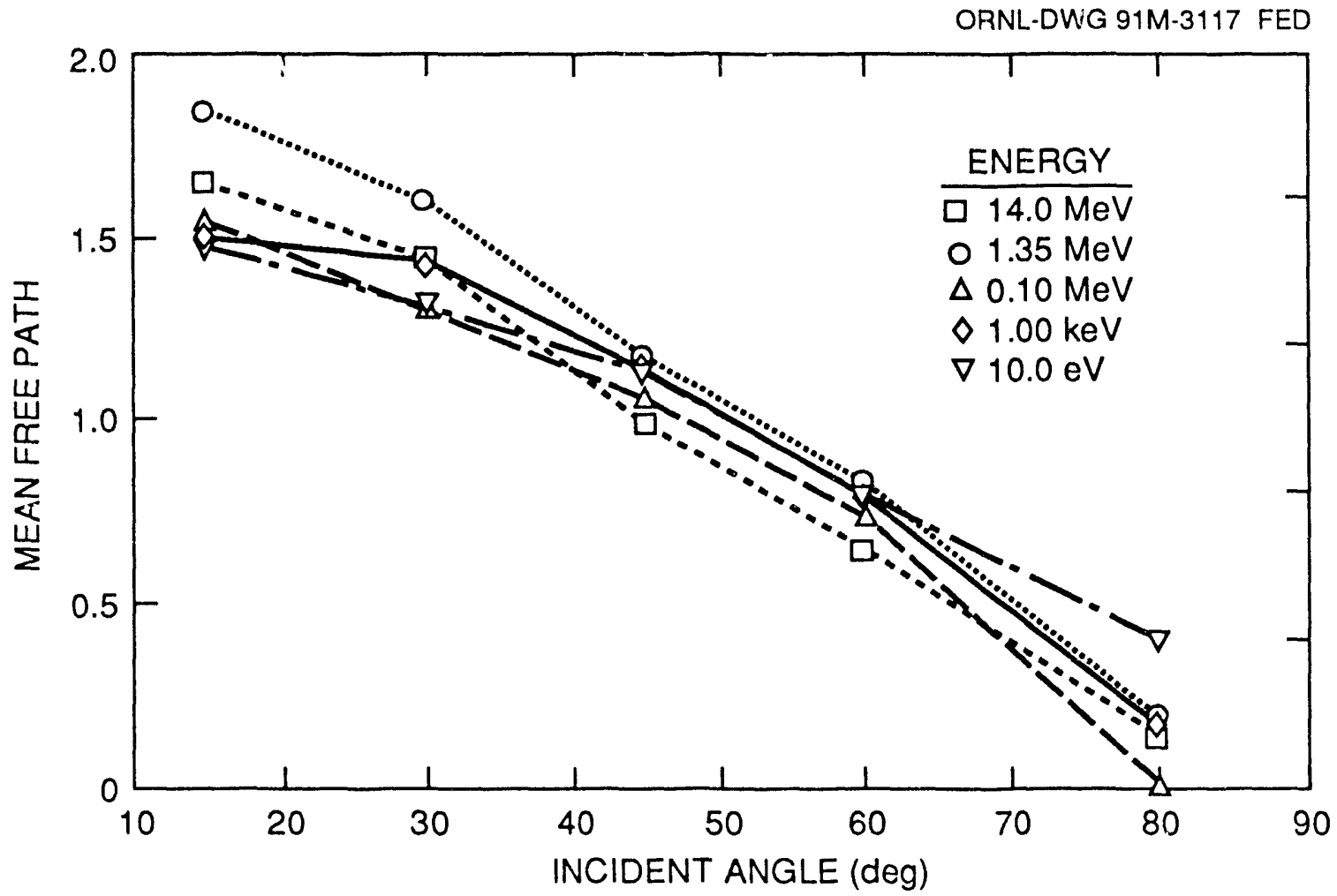

Fig. 4.3. Displacement in number of mean free paths as a function of incident angle for different incident energies for ordinary concrete. 


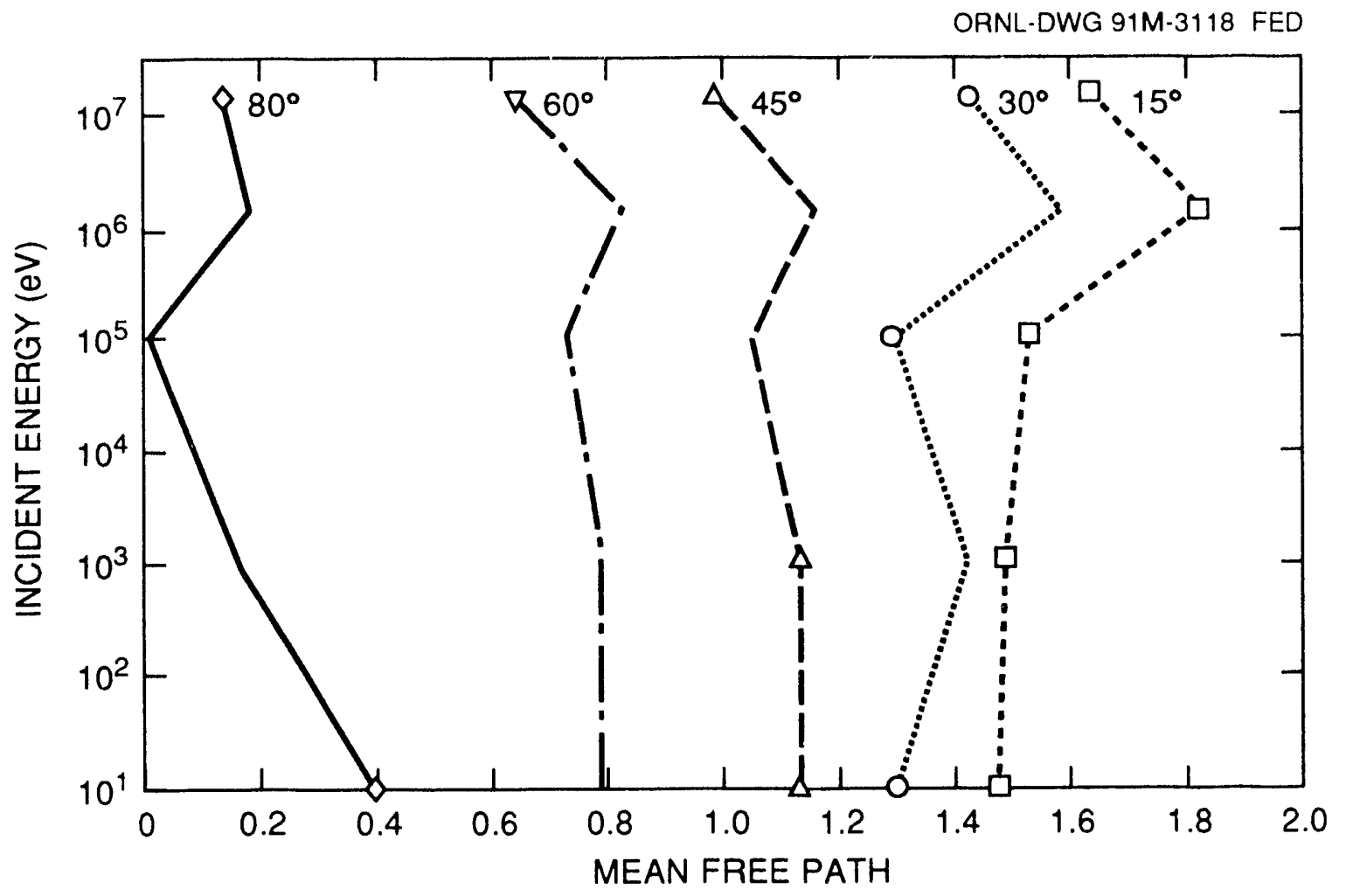

Fig. 4.4. Displacement in number of mean free paths as a function of incident energy for different incident angles for ordinary concrete.

The results in Fig. 4.3 were used to derive a fitting function. The parameters for the function were determined by minimizing the deviations from the calculated points. The derived formula for the displacement of the point of emergence for ordinary concrete is

$$
\rho=0.025\left(90-\Theta_{0}\right) / \Sigma_{T},
$$

where $\rho$ is the displacement in centimeters, $\Theta_{0}$ is the incident angle of the particle measured from the albedo plane, and $\Sigma_{T}$ is the total cross section corresponding to the energy group of the incident particle (reciprocal of the mean free path).

This simplified approach was incorporated into the albedo procedure to demonstrate the influence of the point of emergence on the final results of a calculation. This emergent point represents the average displacement from the point of incidence over all possible outcomes. Symmetry of the diffusion process inside a medium leads to the observation that the displacement vector that defines the so-called average point of emergence must lie in the plane normal to the albedo surface. 


\subsubsection{Implementing the Point of Emergence in the Random Walk}

The MORSE code was modified to make the generated point of emergence information available to the albedo-modified random walk. The fitting function and the particle's incident polar angle were used as described in Sect. 4.2.1 to calculate the average displacement for each albedo collision. The subroutine ALBDO of the MORSE/BREESE code package was modified to accommodate the displacement during the random walk.

A new subroutine, called GOMES, ${ }^{6}$ was written to transfer the particle from the point of incidence to the point of emergence; it also determines the new region and medium numbers at the point of emer jence. The displacement of the point of emergence may result in the creation of an albedo-displaced pseudo-particle inside an albedo region. If this occurs, the emergent particle is banked as if it were a pseudo-particle, and its tracking is resumed with that of the other pseudo-particles after all the source particles have been processed. However, the albedo-displaced pseudo-particle is not killed by any albedo plane and its tracking is performed in a full-transport mode-tracking of the albedo particle that was being followed is terminated as if the albedo particle has been absorbed by the albedo medium, and the next source particle in the bank is processed. As a consequence, up to two pseudo-particles may be created at the same albedo event, one at the point of incidence heading in the same direction as the incident particle and a second at the point of emergence heading in the direction dictated by the albedo reflection process. If the pseudo-particle option is turned off, an emergent particle that is displaced to points inside an albedo region is lilled.

\subsection{INCORPORATION OF SPATIALLY DEPENDENT ALBEDO DATA}

\subsubsection{Generating a New Set of Albedo Data with TORT}

The 3-D discrete ordinates code TORT has an advantage over Monte Carlo codes in providing a detailed clescription of the radiation field in terms of easily accessible discrete ordinates fluxes. To obtain the same level of detail in a Monte Carlo calculation, much more computer time would be required and the calculation would be prohibitively expensive. To calculate albedo data, the boundary angular fluxes are required. The version of the TORT code distributed by RSIC does not have this feature, so it was modified to make this information available.

The TORT code provides several modes for the flux sweep, including the linear or diamond difference model, the step model, and the semianalytical methods (nodal and characteristic). Calculating albedo data is a very difficult problem for discrete ordinate codes because of the punctual, monoenergetic, monodirectional characteristic of the source term. To avoid the propagation or even the occurrence of negative angular fluxes in the results, the available modes were studied to identify 
the best one for generating albedo data. The method that leads to overall positivity of the angular fluxes was the characteristic method, using the highest order of flux expansion available in the code. The diamond difference model and the step model, even with negative flux fix-up, produced negative scalar fluxes. The nodal method did not produce negative scalar fluxes for high-order expansions of the flux, but occasional negative angular fluxes were observed. The characteristic method was the only procedure that exhibited an overall positivity in the scalar and angular fluxes with an acceptable flux shape distribution.

Unfortunately, the characteristic method is very expensive (it requires about three times as much computer time as the diamond difference option for this problem). Given the number of runs required to calculate a set of albedo data, this additional computational expense makes a dramatic difference in the overall cost. However, improvements can be made to specialize the code for efficient albedo generation, and some of these improvements will reduce the required computer time by a factor of two or more.

A set of albedo data was generated for concrete using $S_{10}$ quadrature (145 directions) and a $P_{3}$ set of cross sections with 25 neutron groups and 12 photon groups. A concrete block, $2 \mathrm{~m} \times 2 \mathrm{~m} \times 1.5 \mathrm{~m}$, was modeled with a $13 \times 13 x-y$ spatial mesh and 9 mesh spaces in the direction of the inward normal of the albedo plane.

The directional flux, $N$, was written as it was calculated for each direction, energy, and spatial position on the albedo plane. To transform the directional flux into corresponding albedo data, the relationship

$$
\alpha_{m_{0}, g_{0}, m, i, j, g}=\frac{N_{m, i, j, g} W_{m} \mu_{m}}{S_{m_{0}, i_{0}, j_{0}, g_{0}} W_{m_{0}} A_{i_{0}, j_{0}} \mu_{m_{0}}}
$$

is used. Here $\alpha_{m_{0}, g_{0}, m, i, j, g}$ is the triply differential albedo for particles incident at the quadrature-defined direction $m_{0}$, within the energy group $g_{0}$, which emerge at the quadrature-defined direction $m$, at the spatial mesh point $(i, j)$, and within the energy group $g ; N_{m, i, j, g}$ is the directional flux; $W_{m}$ is the quadrature weight for

direction $m ; \mu_{m}=\cos \Theta ; S_{m_{0}, i_{0}, j_{0}, g_{0}}$ is the source strength; and $A_{i_{0}, j_{0}}$ is the surface area of the spatial mesh into which the source is input.

The value of the albedo for each combination of the incident energy and direction is computed and added to create a joint cumulative probability distribution. The steps needed to manipulate and format the data into forms suitable for use by the modified version of the MORSE code are described in Sect. 4.3.2.

\subsubsection{Processing the Spatially Dependent Albedo Data}

The mechanics of processing and using a set of spatially dependent albedo data differ considerably from those of such a set's 2-D counterpart. The spatial distribution of the points of emergence is added to the albedo data, and the 
symmetry of the angular flux to the plane defined by the incident direction and the normal to the albedo plane no longer holds for the whole domain of the spatial variable.

An interface code called ALBFAC3D was developed to read the TORT files that contain the angular distribution of the emergent flux at the albedo plane and to generate the probability distribution tables. ALBFAC3D writes a file with the albedo information in a format sutable for input into a modified version of the subroutine ALBIN of the MORSE/BREESE package. The marginal-to-conditional probability distribution sampling scheme was changed to accommodate the adjoint mode, which was not procedurally possible with the conventional scheme in the BREESE code. The marginal probability distribution for the reflected albedo particles in the ALBFAC3D code is the energy distribution; the singly conditional probability distribution is for the spatial variable; the doubly conditional probability distribution is for the polar angle; and the triply conditional probability distribution is for the azimuthal probability distribution. This modification in the order of sampling the variables results in a modified normalization procedure through the use of a different scheme of summation. The procedure is implemented in the ALBFAC3D code as follows.

The total albedo for an incident energy $\left(E_{0}^{i_{0}}\right)$ and $\eta$ level $\left(\Theta_{0}^{m_{0}}\right)$ is

$$
\alpha_{\mathrm{T}}\left(E_{0}^{i_{0}}, \Theta_{0}^{m_{0}}\right)=\sum_{i=i_{0}}^{I} \sum_{1}^{J} \sum_{1}^{K} \sum_{1}^{M} \sum_{1}^{N} \alpha\left(E_{0}^{i_{0}}, \Theta_{0}^{m_{0}} ; E_{1}^{i}, X_{j}, Y_{k}, \Theta_{1}^{m}, \phi_{n}\right)
$$

where $E_{0}^{i_{0}}$ and $\Theta_{0}^{m_{0}}$ are the incident energy group and incident $\eta$ level in the quadrature set, respectively. The $i$ th outgoing energy group is $E_{1}^{i}$, and $I$ represents the total number of outgoing energy groups; $X_{j}$ and $Y_{k}$ are displacement components parallel and perpendicular, respectively, to the projection of the incident direction of the particle on the albedo plane, for which $J$ and $K$ are the total numbers of emergent points considered for the parallel and perpendicular components, respectively. The angle $\Theta_{1}^{m}$ represents the $m$ th outgoing $\eta$ level out of a total number $M$ of outgoing $\eta$ levels. The angle $\phi_{n}$ is the $n$th outgoing azimuthal angle out of a maximum number $N$ of azimuthal angles in the quadrature set.

The particle's outgoing energy group from an albedo event is selected according to

$$
\operatorname{cdf}_{E}\left(E_{0}^{i_{0}}, \Theta_{0}^{m_{0}} ; E_{1}^{i_{1}}\right)=\frac{\sum_{i=i_{0}}^{i_{1}} \sum_{1}^{J} \sum_{1}^{K} \sum_{1}^{M} \sum_{1}^{N} \alpha\left(E_{0}^{i_{0}}, \Theta_{0}^{m_{0}} ; E_{1}^{i}, X_{j}, Y_{k}, \Theta_{1}^{m}, \phi_{n}\right)}{\alpha_{\mathrm{T}}\left(E_{0}^{i_{0}}, \Theta_{0}^{m_{0}}\right)},
$$

where $\operatorname{cdf}_{E}$ represents the cumulative marginal probability of a particle being scattered from energy group $i_{0}$ into one of the energy groups $i_{0}$ to $i_{1}$. 
The conditional cdf for the displacement in the direction parallel to the projection of the particle's incident direction on the albedo plane is

$$
\operatorname{cdf}_{X}\left(E_{0}^{i_{0}}, \Theta_{0}^{j_{0}}, E_{1}^{i_{1}} ; X_{j_{1}}\right)=\frac{\sum_{1}^{j_{1}} \sum_{1}^{K} \sum_{1}^{M} \sum_{1}^{N} \alpha\left(E_{0}^{i_{0}}, \Theta_{0}^{m_{0}}, E_{1}^{i} ; X_{j}, Y_{k}, \Theta_{1}^{m}, \phi_{n}\right)}{\sum_{1}^{J} \sum_{1}^{K} \sum_{1}^{M} \sum_{1}^{N} \alpha\left(E_{0}^{i_{0}}, \Theta_{0}^{m_{0}}, E_{1}^{i} ; X_{j}, Y_{k}, \Theta_{1}^{m}, \phi_{n}\right)},
$$

and the cdf for the displacement in the direction perpendicular to the projection of the particle's incident direction on the albedo plane is

$$
\operatorname{cdf}_{Y}\left(E_{0}^{i_{0}}, \Theta_{0}^{j_{0}}, E_{1}^{i_{1}} ; Y_{k_{1}}\right)=\frac{\sum_{1}^{k_{1}} \sum_{1}^{J} \sum_{1}^{M} \sum_{1}^{N} \alpha\left(E_{0}^{i_{0}}, \Theta_{0}^{m_{0}}, E_{1}^{i} ; X_{j}, Y_{k}, \Theta_{1}^{m}, \phi_{n}\right)}{\sum_{1}^{J} \sum_{1}^{K} \sum_{1}^{M} \sum_{1}^{N} \alpha\left(E_{0}^{i_{0}}, \Theta_{0}^{m_{0}}, E_{1}^{i} ; X_{j}, Y_{k}, \Theta_{1}^{m}, \phi_{n}\right)} .
$$

Note that $\operatorname{cdf}_{X}$ and $\operatorname{cdf}_{Y}$ are mutually independent.

The conditional cdf's for the emergent $\eta$ level and emergent azimuthal angle depend on three other variables. The number of discrete values of a variable that must be stored is equal to the number of discrete values of the considered variable multiplied by the matrix of discrete values of all the variables on which the considered variable depends. To minimize the storage requirement for the discrete values of the emergent $\eta$ levels and emergent azimuthal angles, the distributions of these two variables can be collapsed into super-regions of the spatial variables by indicating, through an input parameter to the ALBFAC3D code, the number of spatial mesh points that make up a super-region. In the following formulation for the $\eta$ level and azimuthal angle cdf's, $J K$ denotes the total number of super-regions, $\mathrm{XY}$ denotes the super-region, and $j k$ denotes the index of the super-region. The cdf for the outgoing $\eta$ level is

$$
\operatorname{cdf}_{\Theta}\left(E_{0}^{i_{0}}, \Theta_{0}^{m_{0}}, E_{1}^{i_{1}}, X Y_{j k_{1}} ; \Theta_{1}^{m_{1}}\right)=\frac{\sum_{1}^{m_{1}} \sum_{1}^{N} \alpha\left(E_{0}^{i_{0}}, \Theta_{0}^{m_{0}}, E_{1}^{i}, X Y_{j k_{1}}, \Theta_{1}^{m}, \phi_{n}\right)}{\sum_{1}^{M} \sum_{1}^{N} \alpha\left(E_{0}^{i_{0}}, \Theta_{0}^{m_{0}}, E_{1}^{i}, X Y_{j k_{1}}, \Theta_{1}^{m}, \phi_{n}\right)},
$$

and the cdf for the azimuthal angle is

$$
\operatorname{cdf}_{\phi}\left(E_{0}^{i_{0}}, \Theta_{0}^{j_{0}}, E_{1}^{i_{1}}, X Y_{j k_{1}}, \Theta_{1}^{m_{1}} ; \phi_{n_{1}}\right)=\frac{\sum_{1}^{n_{1}} \alpha\left(E_{0}^{i_{0}}, \Theta_{0}^{m_{0}}, E_{1}^{i}, X Y_{j k_{1}}, \Theta_{1}^{m_{1}}, \phi_{n}\right)}{\sum_{1}^{N} \alpha\left(E_{0}^{i_{0}}, \Theta_{0}^{m_{0}}, E_{1}^{i}, X Y_{j k_{1}}, \Theta_{1}^{m_{1}} ; \phi_{n}\right)} .
$$


The ALBFAC3D code can collapse the number of spatial positions for which the emergent angular distribution is calculated to any number specified by the user. Frr the data to be collasped correctly, the user must provide an input matrix that relates the original mesh space distribution to the super-regions. During the sampling process implemented in the modified version of the subroutine ALBDO, the emergent position ( $j, k$ indexes of the emergent mesh) is sampled before the emergent direction is selected. Then at the time of sampling the emergent direction ALBDO has all the necessary information, which consists of the spatial indices $j$ and $k$ of the emergent mesh and input matrix, to select the emergent direction from the conditional probability distribution for the super-regions. This collapsing capability of the ALBFAC3D code is very useful in reducing the memory space required for the MORSE run, without losing any detail of the description of the point of emergence. The direction distribution is less sensitive to collapsing than the spatial distribution.

To make the MORSE code compatible with the spatially dependent albedo data generated by the ALBFAC3D code, a new version of the subroutine ALBIN was written. The new subroutine, ALBIN3D, allows the user to choose either point or spatially dependent albedos through a new input parameter. The subroutine ALBDO, which performs the albedo reflection process, was also modified and renamed ALBDO3D. The subroutine ALBDO3D allows both forward and adjoint modes of analysis, as discussed in Sect. 5, and is compatible with the albedo data set generated by the modified TORT code and processed by the ALBFAC3D code.

The nodified subroutines and interface codes replace the BREESE system of the MORSE/BREESE package. The new package for formatting, manipulating, and using the spatially dependent albedo data is known as MORSE/STORM.

\subsubsection{Generation of Adjoint Albedo Data}

\subsubsection{Reciprocity relationship for forward and adjuncton albedos}

A reciprocity relationship for the forward and adjuncton albedos was developed to demonstrate that, with proper interpretation and processing, the forward albedo data have a simple relationship with their adjoint counterparts, thereby eliminating the need for separate albedo calculations of the adjuncton albedos. (An adjuncton is a hypothetical particle transported in the $\hat{\Omega} \equiv-\bar{\Omega}$ direction during the generation of random walks in an adjoint Monte Carlo calculation.) The derivation considers a semi-infinite medium with a radiation particle incident on the albedo surface at the spatial position $\overline{\mathbf{r}}_{0}$. This albedo model assumes that the particle emerges from an albedo surface at the spatial position $\overline{\mathbf{r}}_{1}$. In general, albedos calculated with discrete ordinates methods are point albedos rather than the phenomenologically correct spatially dependent a'bedos. The two albedo models are schematically illustrated in Fig. 4.5. 
(a)

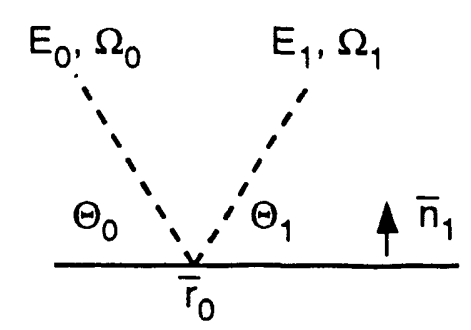

(b)

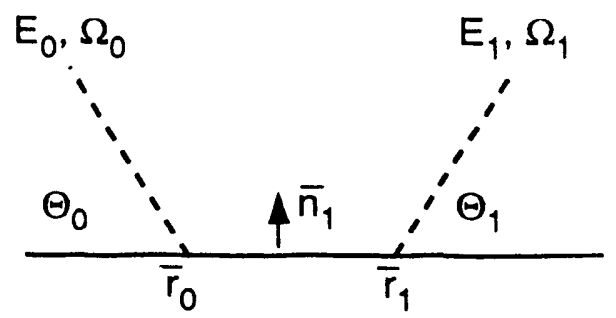

Fig. 4.5. Schematic representation of the reflection process using point and spatially dependent albedo data.

To establish a general reciprocity relationship between the forward and adjuncton albedos, the forward transport equation for the angular flux with phase space coordinates $(\overline{\mathbf{r}}, E, \bar{\Omega})$ resulting from a unit surface source at $\left(\overline{\mathbf{r}}_{0}, E_{0}, \bar{\Omega}_{0}\right)$ is written as

$$
\begin{aligned}
& \overline{\boldsymbol{\Omega}} \cdot \nabla \Phi\left(\overline{\mathbf{r}}_{i} E, \bar{\Omega} ; \overline{\mathbf{r}}_{0}, E_{0}, \bar{\Omega}_{0}\right)+\Sigma_{T}(\overline{\mathbf{r}}, E) \Phi\left(\overline{\mathbf{r}}, E, \bar{\Omega} ; \overline{\mathbf{r}}_{0}, E_{0}, \bar{\Omega}_{0}\right) \\
& =\int_{0}^{\infty} \int_{0}^{4 \pi} d E^{\prime} d \bar{\Omega}^{\prime} \Phi\left(\overline{\mathbf{r}}, E^{\prime}, \bar{\Omega}^{\prime} ; \overline{\mathbf{r}}_{0}, E_{0}, \bar{\Omega}_{0}\right) \Sigma_{s}\left(E^{\prime} \rightarrow E, \bar{\Omega}^{\prime} \rightarrow \bar{\Omega}\right)
\end{aligned}
$$

Similarly, the transport equation for the adjoint flux with phase-space coordinates $(\overline{\mathbf{r}}, E, \bar{\Omega})$ resulting from a unit adjoint surface source at $\left(\overline{\mathbf{r}}_{1}, E_{1}, \bar{\Omega}_{1}\right)$ is written as

$$
\begin{aligned}
& -\bar{\Omega} \cdot \nabla \Phi^{+}\left(\overline{\mathbf{r}}, E, \bar{\Omega} ; \overline{\mathbf{r}}_{1}, E_{1}, \overline{\Omega_{1}}\right)+\Sigma_{T}(\overline{\mathbf{r}}, E) \Phi^{+}\left(\overline{\mathbf{r}}, E, \bar{\Omega} ; \overline{\mathbf{r}}_{1}, E_{1}, \bar{\Omega}_{1}\right) \\
& =\int_{0}^{\infty} \int_{0}^{4 \pi} d E^{\prime} d \bar{\Omega}^{\prime} \Phi^{+}\left(\overline{\mathbf{r}}, E^{\prime}, \bar{\Omega}_{i}^{\prime} ; \overline{\mathbf{r}}_{1}, E_{1}, \bar{\Omega}_{1}\right) \Sigma_{s}\left(E \rightarrow E^{\prime}, \bar{\Omega} \rightarrow \bar{\Omega}^{\prime}\right) .
\end{aligned}
$$

In these formulations, the source ierms are considered external to the system volume and are therefore represented as surface sources. Equation (4.15) is multip.ied by $\Phi^{+}$and Eq. (4.16) by $\Phi$; the resulting equations are subtracted and integrated over the system volume:

$$
\begin{aligned}
& \int_{V}\left(\Phi^{+} \bar{\Omega} \cdot \nabla \Phi+\Phi \bar{\Omega} \cdot \nabla \Phi^{+}\right) d v \\
& =\int_{V} \int_{0}^{\infty} \int_{0}^{4 \pi} d \bar{\Omega}^{\prime} d E^{\prime} d v \Phi^{+}\left(\overline{\mathbf{r}}, E, \bar{\Omega} ; \overline{\mathbf{r}}_{1}, E_{1}, \bar{\Omega}_{1}\right) \Sigma_{s}\left(E^{\prime} \rightarrow E, \bar{\Omega}^{\prime} \rightarrow \bar{\Omega}\right) \\
& \times \Phi\left(\overline{\mathbf{r}}, E^{\prime}, \bar{\Omega}^{\prime} ; \overline{\mathbf{r}}_{0}, E_{0}, \bar{\Omega}_{0}\right)-\int_{V} \int_{0}^{\infty} \int_{0}^{4 \pi} d \bar{\Omega}^{\prime} d E^{\prime} d v \Phi\left(\overline{\mathbf{r}}, E, \bar{\Omega} ; \overline{\mathbf{r}}_{0}, E_{0}, \bar{\Omega}_{0}\right) \\
& \times \Sigma_{s}\left(E \rightarrow E^{\prime}, \bar{\Omega} \rightarrow \bar{\Omega}^{\prime}\right) \Phi^{+}\left(\overline{\mathbf{r}}, E^{\prime}, \bar{\Omega}^{\prime} ; \overline{\mathbf{r}}_{1}, E_{1}, \bar{\Omega}_{1}\right)
\end{aligned}
$$


According to vector calculus,

$$
\int_{V}\left(\Phi^{+} \bar{\Omega} \cdot \nabla \Phi+\Phi \bar{\Omega} \cdot \nabla \Phi^{+}\right) d v=\int_{V} \bar{\Omega} \cdot \nabla \Phi \Phi^{+} d v=\int_{S} \overline{\mathbf{n}} \cdot \bar{\Omega} \Phi \Phi^{+} d s .
$$

By using Eq. (4.18), Eq. (4.17) can be rewritten as

$$
\begin{aligned}
& \int_{S} \overline{\mathbf{n}} \cdot \bar{\Omega} \Phi \Phi^{+} d s \\
& =\int_{V} \int_{0}^{\infty} \int_{0}^{4 \pi} d \bar{\Omega}^{\prime} d E^{\prime} d v\left[\Phi^{+}\left(\overline{\mathbf{r}}, E, \bar{\Omega} ; \overline{\mathbf{r}}_{1}, E_{1}, \bar{\Omega}_{1}\right) \Sigma_{s}\left(E^{\prime} \rightarrow E, \bar{\Omega}^{\prime} \rightarrow \bar{\Omega}\right)\right. \\
& \left.\times \Phi\left(\overline{\mathbf{r}}, E, \bar{\Omega}^{\prime} ; \overline{\mathbf{r}}_{0}, E_{0}, \bar{\Omega}_{0}\right)\right]-\int_{V} \int_{0}^{\infty} \int_{0}^{4 \pi} d \bar{\Omega}^{\prime} d E^{\prime} d v\left[\Phi\left(\overline{\mathbf{r}}, E, \bar{\Omega} ; \overline{\mathbf{r}}_{0}, E_{0}, \bar{\Omega}_{0}\right)\right. \\
& \left.\times \Sigma_{s}\left(E \rightarrow E^{\prime}, \bar{\Omega} \rightarrow \bar{\Omega}^{\prime}\right) \Phi^{+}\left(\overline{\mathbf{r}}, E, \bar{\Omega}^{\prime} ; \overline{\mathbf{r}}_{1}, E_{1}, \bar{\Omega}_{1}\right)\right] .
\end{aligned}
$$

Since the source terms are represented as surface sources, the product $\Phi \Phi^{+}$does not vanish on the surface. The value of $\phi\left(\overline{\mathbf{r}}_{\boldsymbol{s}}\right)$ for $\hat{\mathbf{n}} \cdot \bar{\Omega}<0$ is

$$
\Phi\left(\overline{\mathbf{r}}_{s}\right)_{\overline{\mathbf{n}} \cdot \overline{\mathbf{\Omega}}<0}=\frac{1}{\cos \Theta_{0}} S\left(\overline{\mathbf{r}}_{0}, E_{0}, \bar{\Omega}_{0}\right)=\frac{1}{\left|\overline{\mathbf{n}} \cdot \bar{\Omega}_{0}\right|} \delta\left(\overline{\mathbf{r}}_{s}-\overline{\mathbf{r}}_{0}\right) \delta\left(\bar{\Omega} \cdot \bar{\Omega}_{0}\right) \delta\left(E-E_{0}\right)
$$

Since $\Phi^{+}(\overline{\mathbf{r}})$ is the value of a radiation particle at $\overline{\mathbf{r}}$, the value of $\Phi^{+}(\overline{\mathbf{r}})$ for $\hat{\mathbf{n}} \cdot \bar{\Omega}>0$ is

$$
\Phi_{\dot{\mathbf{n}} \cdot \overline{\mathbf{\Omega}}>0}^{+}=\frac{1}{\cos \Theta_{1}} S^{+}\left(\overline{\mathbf{r}}_{1}, E_{1}, \bar{\Omega}_{1}\right)=\frac{1}{\left|\overline{\mathbf{n}} \cdot \bar{\Omega}_{1}\right|} \delta\left(\overline{\mathbf{r}}_{s}-\overline{\mathbf{r}}_{1}\right) \delta\left(\bar{\Omega} \cdot \bar{\Omega}_{1}\right) \delta\left(E-E_{1}\right)
$$

It follows that

$$
\begin{aligned}
\int_{S} \overline{\mathbf{n}} \cdot \bar{\Omega} \Phi \Phi^{+} d s= & -\int_{S} \delta\left(\overline{\mathbf{r}}_{s}-\overline{\mathbf{r}}_{0}\right) \delta\left(\bar{\Omega} \cdot \bar{\Omega}_{0}\right) \delta\left(E-E_{0}\right) \Phi^{+} d s \\
& +\int_{S} \delta\left(\overline{\mathbf{r}}_{s}-\overline{\mathbf{r}}_{1}\right) \delta\left(\bar{\Omega} \cdot \bar{\Omega}_{1}\right) \delta\left(E-E_{1}\right) \Phi d s \\
= & -\delta\left(\bar{\Omega} \cdot \bar{\Omega}_{0}\right) \delta\left(E-E_{0}\right) \Phi^{+}\left(\overline{\mathbf{r}}_{0}, E, \bar{\Omega} ; \overline{\mathbf{r}}_{1}, E_{1}, \bar{\Omega}_{1}\right) \\
& +\delta\left(\bar{\Omega} \cdot \bar{\Omega}_{1}\right) \delta\left(E-E_{1}\right) \Phi\left(\overline{\mathbf{r}}_{1}, E, \bar{\Omega} ; \overline{\mathbf{r}}_{0}, E_{0}, \bar{\Omega}_{0}\right)
\end{aligned}
$$

Substituting Eq. (4.22) into Eq. (4.17) and integrating over energy and solid angle yields

$$
\begin{aligned}
& -\int_{0}^{\infty} \int_{0}^{4 \pi} d \bar{\Omega} d E \delta\left(\bar{\Omega} \cdot \bar{\Omega}_{0}\right) \delta\left(E-E_{0}\right) \Phi^{+}\left(\overline{\mathbf{r}}_{0}, E, \bar{\Omega} ; \overline{\mathbf{r}}_{1}, E_{1}, \bar{\Omega}_{1}\right) \\
& \quad+\int_{0}^{\infty} \int_{0}^{4 \pi} d \bar{\Omega} d E \delta\left(\bar{\Omega} \cdot \bar{\Omega}_{1}\right) \delta\left(E-E_{1}\right) \Phi\left(\overline{\mathbf{r}}_{0}, E, \bar{\Omega} ; \overline{\mathbf{r}}_{0}, E_{0}, \bar{\Omega}_{0}\right)
\end{aligned}
$$




$$
\begin{aligned}
=\int_{V} & \int_{0}^{\infty} \int_{0}^{4 \pi} \int_{0}^{\infty} \int_{0}^{4 \pi} d \bar{\Omega}^{\prime} d E^{\prime} d \bar{\Omega} d E d v\left\{\left[\Phi^{+}\left(\overline{\mathbf{r}}, E, \bar{\Omega} ; \overline{\mathbf{r}}_{1}, E_{1}, \bar{\Omega}_{1}\right)\right.\right. \\
& \left.\times \Sigma_{S}\left(E^{\prime} \rightarrow E, \bar{\Omega}^{\prime} \rightarrow \bar{\Omega}\right) \times \Phi\left(\overline{\mathbf{r}}, E^{\prime}, \bar{\Omega}^{\prime} ; \overline{\mathbf{r}}_{0}, E_{0}, \bar{\Omega}_{0}\right)\right] \\
& -\left[\Phi\left(\hat{\mathbf{r}}, E^{\prime}, \bar{\Omega}^{\prime} ; \hat{\mathbf{r}}_{0}, E_{0}, \bar{\Omega}_{0}\right) \Sigma_{s}\left(E^{\prime} \rightarrow E, \bar{\Omega}^{\prime} \rightarrow \bar{\Omega}\right)\right. \\
& \left.\left.\times \Phi^{+}\left(\overline{\mathbf{r}}, E, \bar{\Omega} ; \overline{\mathbf{r}}_{1}, E_{1}, \bar{\Omega}_{1}\right)\right]\right\}
\end{aligned}
$$

The last integral in Eq. (4.23) is identically zero, and evaluating the energy and solid-angle integrals results in the relationship

$$
\Phi\left(\overline{\mathbf{r}}_{1}, E_{1}, \bar{\Omega}_{1} ; \overline{\mathbf{r}}_{0}, E_{0}, \bar{\Omega}_{0}\right)=\Phi^{+}\left(\overline{\mathbf{r}}_{0}, E_{0}, \bar{\Omega}_{0} ; \overline{\mathbf{r}}_{1}, E_{1}, \bar{\Omega}_{1}\right)
$$

The $\Phi$ term in Eq. (4.24) is usually interpreted physically as a differential albedo that describes the reflected angular flux associated with an incident unit current; that is, $\Phi\left(\overline{\mathbf{r}}_{1}, E_{1}, \bar{\Omega}_{1} ; \overline{\mathbf{r}}_{0}, E_{0}, \bar{\Omega}_{0}\right) d P_{1}$ is the reflected angular flux in $d P_{1}$ about $\overline{\mathbf{r}}_{1}, E_{1}, \bar{\Omega}_{1}$ due to a unit source (current) on the albedo surface at $\overline{\mathbf{r}}_{0}, E_{0}, \bar{\Omega}_{0}$.

The $\Phi^{+}$term in Eq. (4.24) may be interpreted as a value or importance function; that is, $\Phi^{+}\left(\overline{\mathbf{r}}_{0}, E_{0}, \bar{\Omega}_{0} ; \overline{\mathbf{r}}_{1}, E_{1}, \bar{\Omega}_{1}\right) d P_{1}$ is the value associated with a particle incident on the albedo surface with phase-space coordinates $\left(\overline{\mathbf{r}}_{0}, E_{0}, \bar{\Omega}_{0}\right)$ that results in a reflected particle of unit ralue that emerges in $d P_{1}$ about $\left(\overline{\mathbf{r}}_{1}, E_{1}, \bar{\Omega}_{1}\right)$.

A more useful interpretation of $\Phi^{+}$is in terms of adjunctons, the hypothetical particles transported in the $\hat{\Omega} \equiv-\bar{\Omega}$ direction during the generation of random walks in an adjoint Monte Carlo calculation. The adjuncton albedo $\hat{\Phi}^{+}$corresponding to $\Phi^{+}$is

$$
\hat{\Phi}^{+}\left(\overline{\mathbf{r}}_{0}, E_{0}, \hat{\Omega}_{0} ; \overline{\mathbf{r}}_{1}, E_{1}, \hat{\Omega}_{1}\right) d P_{0}=\Phi^{+}\left(\overline{\mathbf{r}}_{0}, E_{0},-\bar{\Omega}_{0} ; \overline{\mathbf{r}}_{1}, E_{1},-\bar{\Omega}_{1}\right) d P_{0}
$$

which is the reflected adjuncton angular flux in $d P_{0}$ about $\overline{\mathbf{r}}_{0}, E_{0}, \hat{\Omega}_{0}$ resulting from an incident unit adjuncton current with phase-space coordinates $\overline{\mathbf{r}}_{1}, E_{1}, \hat{\Omega}_{1}$.

With Eq. (4.25), Eq. (4.24) can be rewritten as

$$
\Phi_{a}\left(\overline{\mathbf{r}}_{1}, E_{1}, \Omega_{1} ; \overline{\mathbf{r}}_{0}, E_{0}, \bar{\Omega}_{0}\right)=\hat{\Phi}^{+}\left(\mathbf{r}_{0}, E_{0}, \hat{\Omega}_{0} ; \mathbf{r}_{1}, E_{1}, \hat{\Omega}_{1}\right)
$$

where the subscript a denotes albedos based on emergent angular flux rather than on emergent current. Following the standard convention, the symbol $\alpha$ denctes albedos based on emergent current.

According to Eq. (4.26), the forward angular albedo $\Phi$ that describes the $\left(\overrightarrow{\mathbf{r}}_{0}, E_{0}, \bar{\Omega}_{0} \rightarrow \overline{\mathbf{r}}_{1}, E_{1}, \bar{\Omega}_{1}\right)$ reflection is identical to the adjuncton angular albedo $\hat{\Phi}^{+}$ that describes the $\left(\overline{\mathbf{r}}_{1}, E_{1}, \hat{\Omega}_{1} \rightarrow \overline{\mathbf{r}}_{0}, E_{0}, \hat{\Omega}_{0}\right)$ reflection. Therefore, a transformation of the forward angular flux data to create the adjuncton albedos should be possible; the direct calculation of the adjuncton angular albedos $\Phi^{+}$should not be required. The format of the forward angular albedo data is similar to that of the differential scattering cross-section data, and a similar transpose of the forward angular albedo $\Phi_{a}$ data during processing creates the $\alpha^{+}$data set used during the reflection of 
adjunctons. Certain derived quantities such as the total albedos and the cdf's for selection of reflected energy group and direction are also calculated. These quantities are necessarily different from their forward counterparts because the various summations required during normalization procedures are over the $\left(E_{0}, \bar{\Omega}_{0}\right)$ space rather than over the $\left(E_{1}, \bar{\Omega}_{1}\right)$ space.

\subsubsection{Generation procedure and data manipulation}

A standard input option for generating adjoint albedo data is included in ALBFAC3D. The calculation of the total adjuncton albedo is similar to that of its forward counterpart, but the indexes in the summation over $i$ are modified to correctly describe the flow of an adjoint calculation, giving, as a result, an $\alpha_{\mathrm{T}}^{+}$ that is different from $\alpha_{\mathrm{T}}$. The adjoint total albedo is calculated by a procedure in ALBFAC3D as

$$
\alpha_{\mathrm{T}}^{+}\left(E_{1}^{i_{1}}, \Theta_{1}^{m_{1}}\right)=\sum_{i=1}^{i_{1}} \sum_{1}^{J} \sum_{1}^{K} \sum_{1}^{M} \sum_{1}^{N} \alpha\left(E_{0}^{i}, \Theta_{0}^{m} ; E_{1}^{i_{1}}, X_{j}, Y_{k}, \Theta_{1}^{m_{1}}, \phi_{n}\right) .
$$

The summation is performed over the incident energy groups and polar angles. The index for the azimuthal angle varies as it does for the forward mode.

The outgoing energy of an adjuncton experiencing an albedo event is very similar to that of its forward counterpart. The most important difference is that adjunctons scatter upward in energy - that is, from low energy groups to a higher energy group. The cumulative distribution table for adjuncton albedo energy reflects this difference. The forward angular flux generated by a discrete ordinates code is used for generating the probability table, but the indices for the summation are modified to reflect the backward flow of the adjunctons. In ALBFAC3D, the cdf for an adjuncton's outgoing energy is

$$
\operatorname{cdf}_{E}^{+}\left(E_{1}^{i_{1}}, \Theta_{1}^{m_{1}} ; E_{0}^{i_{0}}\right)=\frac{\sum_{i=i_{0}}^{i_{1}} \sum_{1}^{J} \sum_{1}^{K} \sum_{1}^{M} \sum_{1}^{N} \alpha\left(E_{0}^{i_{0}}, \Theta_{1}^{m_{1}} ; E_{1}^{i_{1}}, X_{j}, Y_{k}, \Theta_{1}^{m_{1}}, \phi_{n}\right)}{\alpha_{\mathrm{T}}^{+}\left(E_{1}^{i_{1}}, \Theta_{1}^{m_{1}}\right)} .
$$

The energy index $i_{1}$, which is the upper limit for the summation performed over the energy groups, is fixed, and the index $i_{0}$, which is the lower limit of the summation, varies. The summation over the $\eta$ level, represented by the variables $\Theta_{1}^{m_{1}}$ and $\Theta_{0}^{m}$, is performed in reverse order, so that it is over the incoming $\eta$ level and not over the outgoing $\eta$ level. 
The adjoint displacement $\mathrm{cdf}$ is calculated as

$$
\operatorname{cdf}_{X}^{+}\left(E_{1}^{i_{1}}, \Theta_{1}^{m_{1}}, E_{0}^{i_{0}} ; X_{j_{0}}\right)=\frac{\sum_{1}^{j_{1}} \sum_{1}^{K} \sum_{1}^{M} \sum_{1}^{N} \alpha\left(E_{0}^{i_{0}}, \Theta_{0}^{m}, E_{1}^{i_{1}} ; X_{j}, Y_{k}, \Theta_{1}^{m_{1}}, \phi_{n}\right)}{\sum_{1}^{J} \sum_{1}^{K} \sum_{1}^{M} \sum_{1}^{N} \alpha\left(E_{0}^{i_{0}}, \Theta_{0}^{m}, E_{1}^{i_{1}} ; X_{j}, Y_{k}, \Theta_{1}^{m_{1}}, \phi_{n}\right)}
$$

where the same modification of indices is performed, and the displacement selection procedure is performed for the adjuncton as it would be for a forward particle. The procedure for $\operatorname{cdf}_{Y}^{+}$is the same.

The cdf to select the outgoing $\eta$ level is

$$
\operatorname{cdf}_{\Theta}^{+}\left(E_{1}^{i_{1}}, \Theta_{1}^{m_{1}}, E_{0}^{i_{0}}, X Y_{j k_{0}} ; \Theta_{0}^{m_{0}}\right)=\frac{\sum_{1}^{m_{0}} \sum_{1}^{N} \alpha\left(E_{0}^{i_{0}}, \Theta_{0}^{m}, E_{1}^{i_{1}}, X Y_{j k_{0}}, \Theta_{1}^{m_{1}}, \phi_{n}\right)}{\sum_{1}^{M} \sum_{1}^{N} \alpha\left(E_{0}^{i_{0}}, \Theta_{0}^{m}, E_{1}^{i_{1}}, X Y_{j k_{0}}, \Theta_{1}^{m_{1}}, \phi_{n}\right)},
$$

and the cdf for the azimuthal angle is

$$
\operatorname{cdf}_{\phi}^{+}\left(E_{1}^{i_{1}}, \Theta_{1}^{m_{1}}, E_{0}^{i_{0}}, X Y_{j k_{0}}, \Theta_{0}^{m_{0}} ; \phi_{n_{0}}\right)=\frac{\sum_{1}^{n_{0}} \alpha\left(E_{0}^{i_{0}}, \Theta_{0}^{m_{0}}, E_{1}^{i}, X Y_{j k_{1}}, \Theta_{1}^{m_{1}}, \phi_{n}\right)}{\sum_{1}^{N} \alpha\left(E_{0}^{i_{0}}, \Theta_{0}^{m_{0}}, E_{1}^{i}, X Y_{j k_{1}}, \Theta_{1}^{m_{1}} ; \phi_{n}\right)} .
$$

It is easy to see that the cumulative probability distribution for the adjuncton is exactly the same as for its forward counterpart, except that the angles have opposite signs because the adjuncton's direction is opposite to that of its forward counterpart. A schematic representation of the azimuthal reflection process is presented in Fig. 4.6. The incident direction for the forward mode is the emergent direction for the adjoint mode. The probability of reflection between the two directions remains the same (for both forward and adjoint modes) as stated earlier, but the direction of measuring the angles is inverted. This has no effect on the polar angle, which is measured from the normal to the plane, but it does have an effect on the sign of the azimuthal angle.

\subsubsection{Normalization Factors for Adjoint Calculations}

The MORSE code is designed to perform adjoint calculations in much the same way as forward calculations. The input instructions and the cross-section sets are given in the forward format, and the code makes the necessary inversions. 
ORNL-DWG 91M-3120 FED

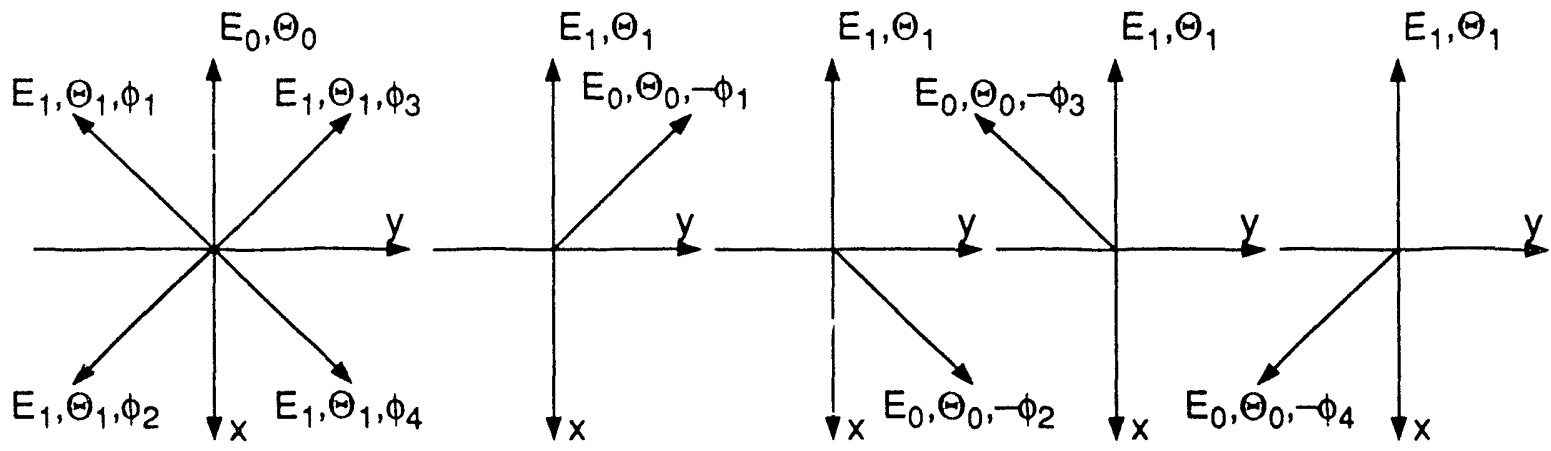

Fig. 4.6. Forward and adjoint azimuthal albedo distributions for fixed incident energy and angle and for fixed outgoing energy and polar angle.

Adjoint source and estimation procedures that can be described by standard input parameters produce responses that are intrinsically made compatible with their forward counterparts by the code. If estimation or source routines are provided by the user, a careful analysis is needed to ensure that the adjoint responses are correctly normalized. Using adjuncton-albedo data in a Monte Carlo adjoint mode of analysis requires no additional normalization factors, and the same normalization procedures used for a normal adjoint calculation can be applied.

The Monte Carlo estimate of the effect of interest $\lambda$ for the forward mode is given by the functional

$$
\lambda_{\text {for }}=\int_{E} \int_{\overline{\mathbf{r}}} \int_{\dot{\Omega}} P^{\Phi}(\overline{\mathbf{r}}, E, \bar{\Omega}) \Phi(\overline{\mathbf{r}}, E, \bar{\Omega}) d \overline{\mathbf{r}} d E d \bar{\Omega}
$$

where $P^{\Phi}(\overline{\mathbf{r}}, E, \bar{\Omega})$ is the response function of $\lambda$ due to a unit angular flux with direction $\bar{\Omega}$, energy $E$, and position $\overline{\mathbf{r}}$, and $\Phi(\overline{\mathbf{r}}, E, \bar{\Omega})$ is the time-independent angular flux. This functional is automatically evaluated by MORSE, and the result is normalized to provide an answer in terms of a unit source particle. The total effect of interest is then determined by multiplying the Monte Carlo estimate for the effect of interest by the strength of the source term.

In the adjoint mode, the relationship between the adjuncton angular flux $\chi^{*}(\overline{\mathbf{r}}, E, \bar{\Omega})$ and the adjoint response function $S(\overline{\mathbf{r}}, E, \bar{\Omega})$ is

$$
\lambda_{\text {adj }}=\int_{E} \int_{\dot{\mathbf{r}}} \int_{\boldsymbol{\Omega}} S(\overline{\mathbf{r}}, E, \hat{\mathbf{\Omega}}) \chi^{*}(\overline{\mathbf{r}}, E, \hat{\mathbf{\Omega}}) d \overline{\mathbf{r}} d E d \hat{\mathbf{\Omega}}
$$

where $S(\overline{\mathbf{r}}, E, \hat{\Omega})$ is the forward source ierm with direction $\hat{\Omega}$, energy $E$, and position $\overline{\mathbf{r}}$, and $\chi^{*}(\overline{\mathbf{r}}, E, \hat{\Omega})$ is the time-independent adjoint angular flux due to the adjoint source term $P^{\Phi}(\overline{\mathbf{r}} . E, \hat{\Omega})$. 
Equation (4.33) can be rewritten in terms of energy groups by replacing the integral over the energy with a summation over the energy groups,

$$
\lambda_{\text {adj }}=\sum_{g=1}^{G} \int_{\overline{\mathbf{r}}} \int_{\bar{\Omega}} S_{g}(\overline{\mathbf{r}}, \hat{\boldsymbol{\Omega}}) \chi_{g}^{*}(\overline{\mathbf{r}}, \hat{\boldsymbol{\Omega}}) d \overline{\mathbf{r}} d \hat{\mathbf{\Omega}}
$$

where $S_{g}(\overline{\mathbf{r}}, \hat{\Omega})$ is the forward source term for energy group $g$, with direction $\hat{\Omega}$ and position $\overline{\mathbf{r}}$, and $\chi_{g}^{*}(\overline{\mathbf{r}}, \hat{\Omega})$ is the time-independent adjuncton angular flux for the energy group $g$, with direction $\hat{\Omega}$ and position $\overline{\mathbf{r}}$.

The Monte Carlo estimate for $\lambda$ should produce exactly the same values for both the forward and the adjoint modes of analysis, but because of the code normalization procedures, this may not be true for all cases.

To evaluate the normalization factors that the code includes in the final answer and additional factors that should be applied by the user, it is important to perform a step-by-step analysis of the normalization procedure. Accordingly, brief descriptions of the units and the nature of the physical variables used in evaluating the functionals are presented.

In Eq. (4.32), the response function $P^{\Phi}$ is a value function and as such carries the units of response per unit fluence. The units of the effect of interest for the forward mode are the response associated with $P^{\Phi}$.

The source term of the forward Monte Carlo mode of analysis is a differential distribution,

$$
S_{\mathrm{for}}=S(\overline{\mathbf{r}}, E, \overline{\mathbf{\Omega}})
$$

When the functional of the effect of interest in the adjoint mode is evaluated, the same normalization procedures used in the forward mode are applied by the code (except for the energy, which is discussed later). The functional for the effect of interest in the adjoint mode is evaluated [Eq. (4.29)] using the adjuncton angular flux and the adjoint response function, which is the forward source term. It is well known that the adjoint flux is not a differential distribution and has the units of response per unit particle.

MORSE executes the same procedures for an adjoint mode as for the forward mode. The normalization factor applied to the adjoint source is

$$
N_{\mathrm{adj}}^{s}=\int S^{*}(P) d P
$$

This follows because MORSE is designed to normalize the source term to unity regardless of units. As an example, consider a point adjoint source (which corresponds to a point detector in the forward mode) for which the forward group response functions are set equal to unity (isotropic flux detector) for $G$ energy groups. In this case the adjoint source normalization factor is 


$$
\begin{aligned}
N_{\mathrm{adj}}^{s} & =\int P^{\Phi}(E, \bar{\Omega}) d E d \bar{\Omega}=\sum \int_{4 \pi} d \bar{\Omega} \\
& =4 \pi G
\end{aligned}
$$

As a consequence, the results of an adjoint calculation must be multiplied by the factor $4 \pi G$. The user enters the source energy spectrum with the E1 card in the MORSE input file, and the code automatically multiplies the Monte Carlo estimate by the summation of the values entered by the E1 card. In this example, this summation equals $G$.

Therefore, the adjoint estimate can be made to yield essentially the same answer as the forward calculation (response per unit source particle) if the adjoint estimate is divided by the source normalization factor,

$$
N_{\mathrm{adj}}^{d}=\int R_{\mathrm{adj}}(P) d P .
$$

In the adjoint mode, MORSE sees the forward source $S(P)$ as a response function $R_{\mathrm{adj}}(P)$ and does not automatically normalize it. As an example, consider a forward isotropic point source defined over $N$ energy groups, which in the forward case has a uniform probability of being sampled. Then Eq. (4.38) can be evaluated as

$$
\begin{aligned}
N_{\mathrm{adj}}^{d} & =\int R_{\mathrm{adj}}(P) \delta\left(\overline{\mathbf{r}}-\overline{\mathbf{r}}_{0}\right) d P=\int_{E} \int_{4 \pi} R_{\mathrm{adj}}\left(E^{\prime}, \bar{\Omega}\right) d E^{\prime} d \vec{\Omega} \\
& =\sum_{n=1}^{N} \int_{4 \pi} d \Omega=4 \pi N
\end{aligned}
$$

and the $4 \pi N$ normalizaton factor for the forward source does in fact multiply the adjoint estimate. Therefore, the adjoint MORSE Monte Carlo answer must be divided by this factor. In this example the $4 \pi$ factors cancel out, but this may not be the case for different calculations.

\section{RESULTS}

\subsection{THE STANDARD MORSE/BREESE PACKAGE}

With the MORSE/BREESE package, the MORSE Monte Carlo random walk is modified by the albedo event such that particle transport is not performed within albedo media. Next-event estimation is performed at each albedo event; the probability that a particle will be reflected in the direction of the detector is multiplied by the probability for uncollided transport of the particle to the detector. The standard MORSE/BREESE package makes contributions only to detectors in the hemisphere of the incident particle. This means that certain 
potentially very important contributions from other albedo media cannot be included. This assumption might produce acceptable results if the effect of interest is not measurably affected by contributions from collisions inside the albedo region. It therefore follows that a careful use of the next-event estimator in conjunction with albedo-modified random walks is required.

The boundary-crossing estimator receives contributions from particles that actually cross designated surfaces and does not allow estimation at positions other than those enclosed by albedo surfaces.

The shield configuration shown in Figs. 5.1 and 5.2 was studied to evaluate the performance of both estimators. The concrete composition used and the energy group structure of the cross sections are presented in Ref. 6. Monte Carlo solutions were obtained for each type of estimator and compared with full-transport MORSE and MCNP benchmarks for this problem. Table 5.1 presents the results, the fractional standard deviations, and the figures of merit for each case. The MORSE/BREESE calculations underestimate the correct answer, even though the fractional standard deviations are relatively small. The next-event and boundarycrossing estimators do not yield the same results, and the standard deviation for the boundary-crossing estimator is large even after 5,000,000 particles have been

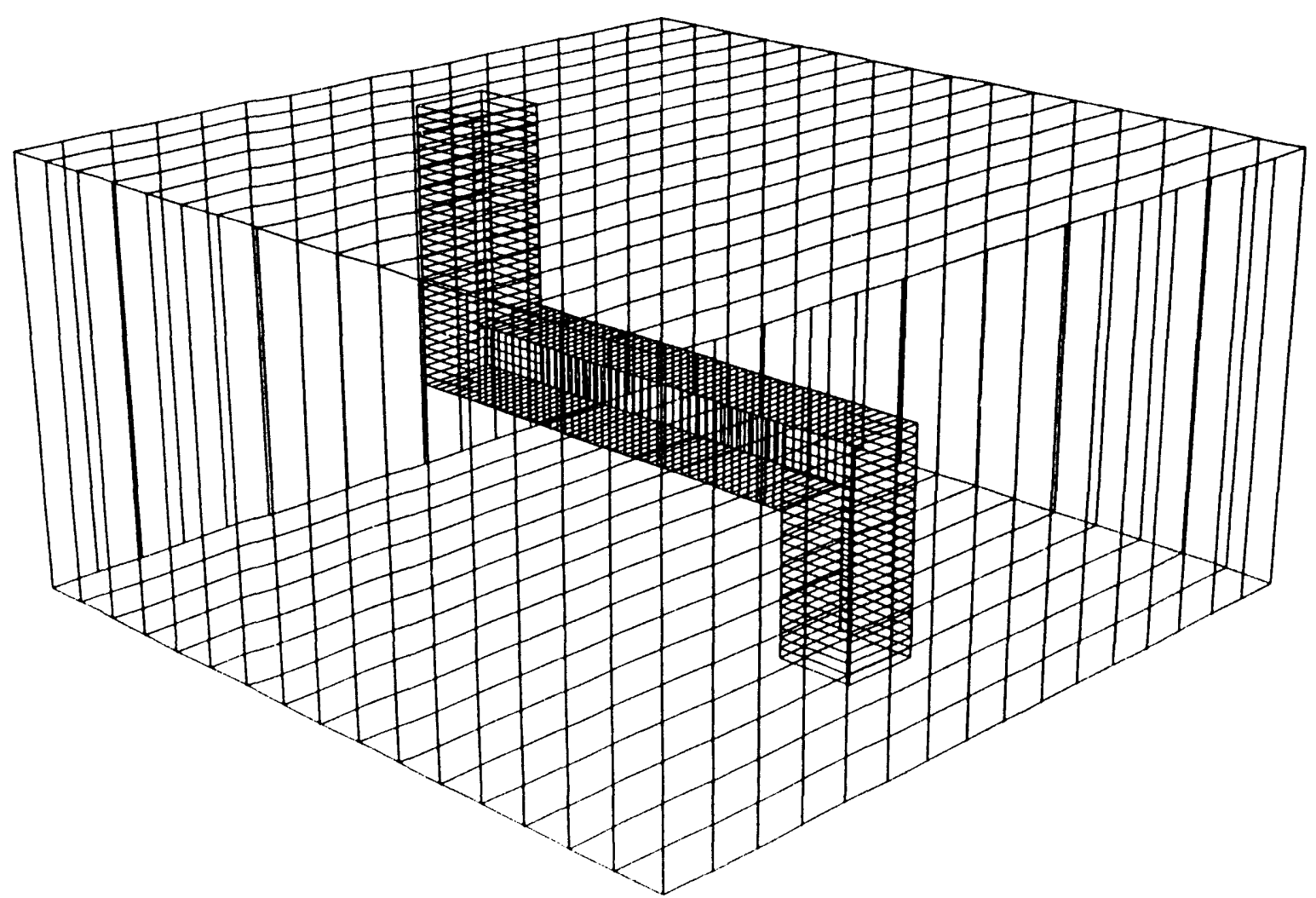

Fig. 5.1. Three-dimensional view of the geometry of the sample problem. 
ORNL-DWG $91 M-3122$ FED

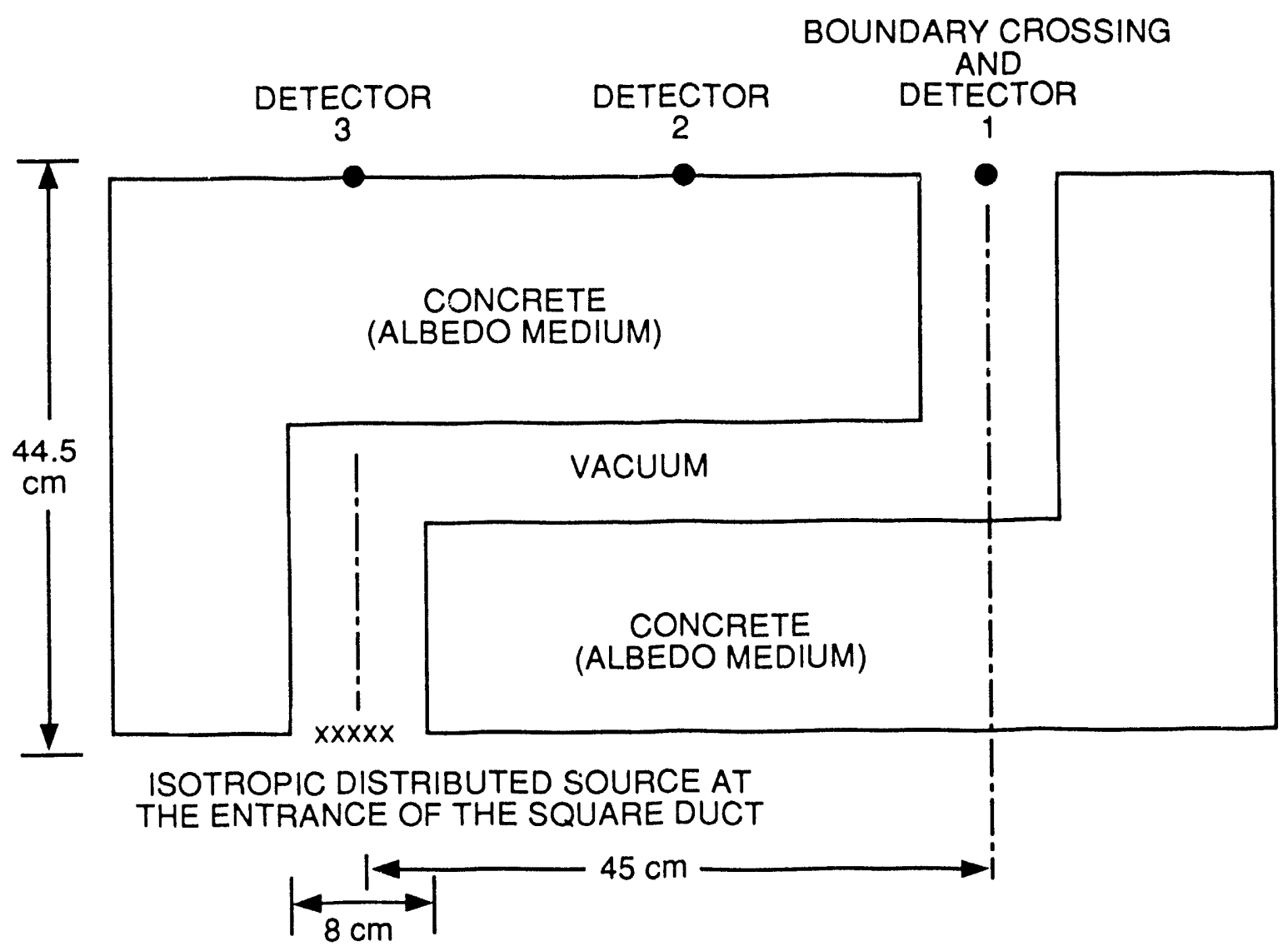

Fig. 5.2. Shield configuration and detector locations for the sample problem.

processed. Clearly, the MORSE/BREESE code package does not faithfully model the sample problem.

The problem solved presents a complex geometry: a three-legged streaming path for the radiation and a small physical size. The traditional "corner effect" could lead to the large discrepancies observed in the results, because the albedos calculated for semi-infinite media do not correctly represent the full-transport behavior near the intersections of albedo surfaces. Nonphysical distortions of the radiation field occur when two adjacent albedo surfaces intersect to form a corner. Calculations were performed with full transport within a concrete wedge at each corner to determine whether this would mitigate the problem. The results are shown in Table 5.2. These results are much closer to the benchmark results than those calculated without full transport at the corners. The next-event estimator detector and the boundarycrossing estimator detector located at the exit of the duct had significant gains with full transport at the corners. However, the results are still significantly lower than the benchmark answers. Detectors at other positions did not demonstrate the same 
Table 5.1. Results for benchmark cases (full-transport MORSE and MCNP) and for the standard MORSE/BREESE package using next-event and boundary-crossing estimators

\begin{tabular}{|c|c|c|c|c|}
\hline & \multicolumn{4}{|c|}{ Code } \\
\hline & \multirow[b]{2}{*}{ MORSE } & \multirow[b]{2}{*}{ MCNP } & \multicolumn{2}{|c|}{ MORSE/BREESE } \\
\hline & & & Next-event & $\begin{array}{c}\text { Boundary- } \\
\text { crossing }\end{array}$ \\
\hline $\begin{array}{l}\text { Source particles } \\
\text { processed }\end{array}$ & 500,000 & $3,000,000$ & $1,000,000$ & $5,000,000$ \\
\hline \multicolumn{5}{|l|}{ Total flux ${ }^{a}$} \\
\hline Detector 1 & $1.0618 \times 10^{-5}$ & $1.1549 \times 10^{-5}$ & $3.9303 \times 10^{-7}$ & $5.8597 \times 10^{-8}$ \\
\hline Detector 2 & $3.3060 \times 10^{-5}$ & $3.30359 \times 10^{-5}$ & $2.0154 \times 10^{-6}$ & - \\
\hline Detector 3 & $6.8373 \times 10^{-5}$ & $7.35721 \times 10^{-5}$ & $8.1034 \times 10^{-6}$ & - \\
\hline \multicolumn{5}{|l|}{$\begin{array}{l}\text { Fractional stand- } \\
\text { ard deviation }\end{array}$} \\
\hline Detector 1 & 0.0161 & 0.0267 & 0.0013 & 0.12015 \\
\hline Detector 2 & 0.0310 & 0.0272 & 0.0034 & - \\
\hline Detector 3 & 0.0240 & C 0330 & 0.00001 & - \\
\hline \multicolumn{5}{|l|}{ Figure of merit $b$} \\
\hline Detector 1 & 3.67 & - & 17,584 & 1.19 \\
\hline Detector 2 & 0.99 & - & 2,571 & - \\
\hline Detector 3 & 1.65 & - & $2.97 \times 10^{8}$ & - \\
\hline
\end{tabular}

aUnits: neutrons $\cdot \mathrm{cm}^{-2} \cdot(\text { source neutrons })^{-1} \cdot \mathrm{s}^{-1}$.

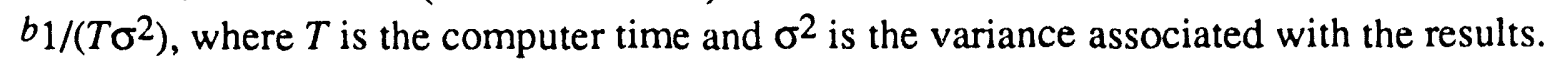

improvements. The problem of estimating to detectors outside the albedo surface envelope remained unresolved, primarily because of the very restricted nature of the albedo statistical estimation procedure.

\subsection{THE PSEUDO-PARTICLE MODIFICATION}

Tables 5.3 and 5.4 present results for the sample problem of Sect. 5.1, calculated with the modified version of the MORSE/BREESE code package that creates and tracks pseudo-particles inside albedo regions. No biasing technique was applied when tracking pseudo-particles or albedo particles in a full-transport mode at the corners (Table 5.4). However, the survival biasing techniques described in Sect. 4.1.1 
Table 5.2. Results for benchmark case (full-transport MORSE) and for the MORSE/BREESE package using next-event and boundarycrossing estimators with full transport at the corners

\begin{tabular}{lccc}
\hline & & \multicolumn{3}{c}{ Code } \\
\cline { 2 - 4 } & MORSE & Next-event & $\begin{array}{c}\text { Boundary- } \\
\text { crossing }\end{array}$ \\
\cline { 2 - 4 } & 500,000 & 500,000 & $1,000,000$ \\
\hline Source particles & & & \\
processed & & & \\
Total flux & & & \\
Detector 1 & $1.0618 \times 10^{-5}$ & $2.7672 \times 10^{-6}$ & $8.6265 \times 10^{-7}$ \\
Detector 2 & $3.3060 \times 10^{-5}$ & $4.4297 \times 10^{-6}$ & - \\
Detector 3 & $6.8373 \times 10^{-5}$ & $1.1727 \times 10^{-5}$ & - \\
Fractional stand- & & & \\
ard deviation & & 0.0462 & 0.1195 \\
Detector 1 & 0.0161 & 0.00453 & - \\
Detector 2 & 0.0310 & 0.00179 & - \\
Detector 3 & 0.0240 & & - \\
Figure of merit $b$ & & 4.18 & - \\
Detector 1 & 3.67 & 435 & - \\
Detector 2 & 0.99 & 2786 & - \\
Detector 3 & 1.65 & &
\end{tabular}

aUnits: neutrons $\mathrm{cm}^{-2} \cdot(\text { source neutrons) })^{-1} \mathrm{e}^{-1}$.

$b_{1} /\left(T \sigma^{2}\right)$, where $T$ is the computer time and $\sigma^{2}$ is the variance associated with the results.

were used in the generation of pseudo-particles. In Tables 5.3 and 5.4, case 1 has no biasing. For case 2, the average weight of the albedo-transported particles was used for biasing the generation of yseudo-particles; for case 3, cosine biasing; and for case 4, probabilities assigned by energy group and by geometry region.

In Table 5.3, significant improvements are evident for all detectors, in particular for those located behind albedo surfaces (detectors 2 and 3 ). The cosine biasing scheme was found to be the best option for this problem. Assigning probabilities by energy group and region might have been more effective if the input probabilities had been based on adjoint information.

The calculations for the cases in Table 5.4 were performed with full transport at the corners. The results for detector 1 are about $50 \%$ lower than those without 


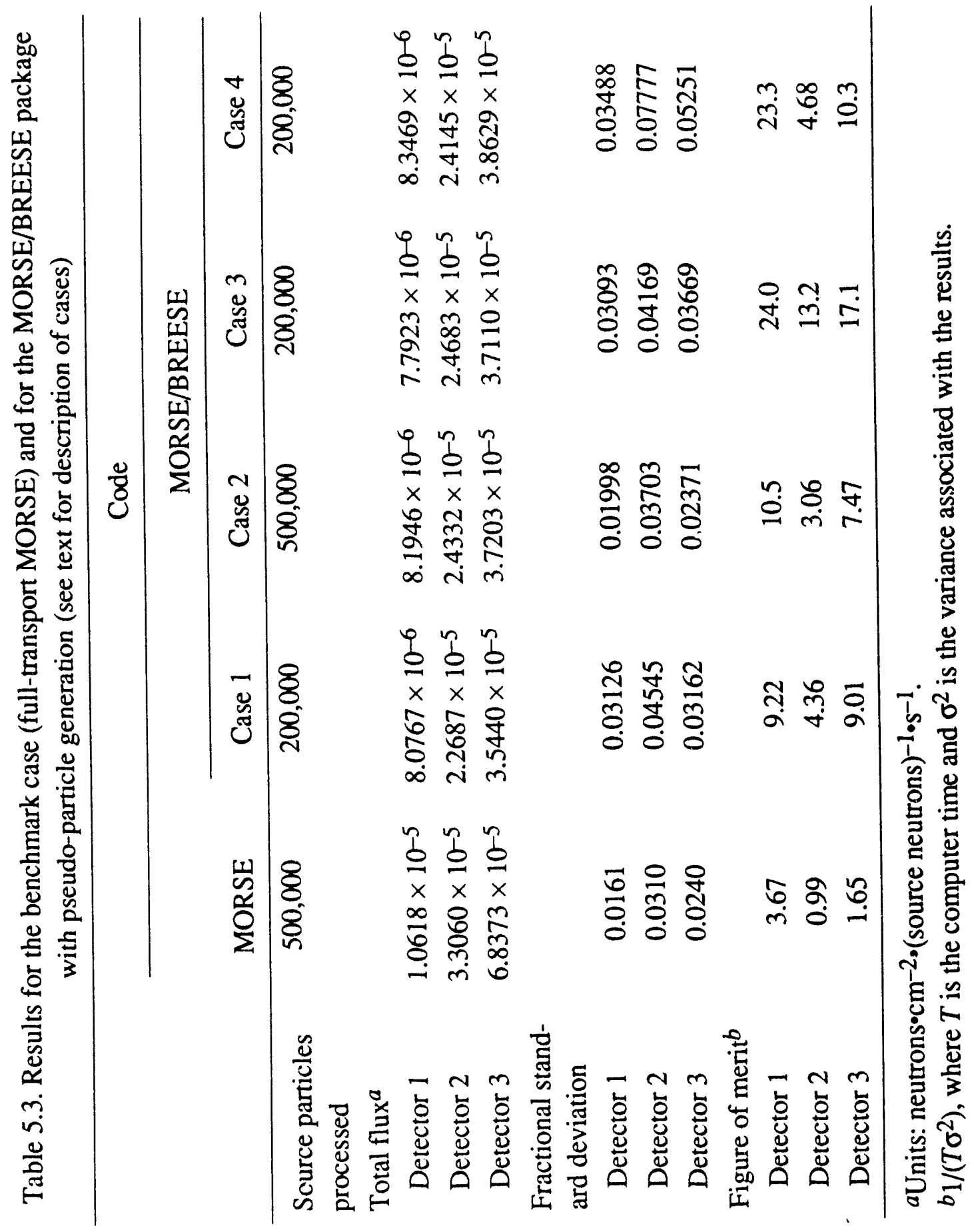




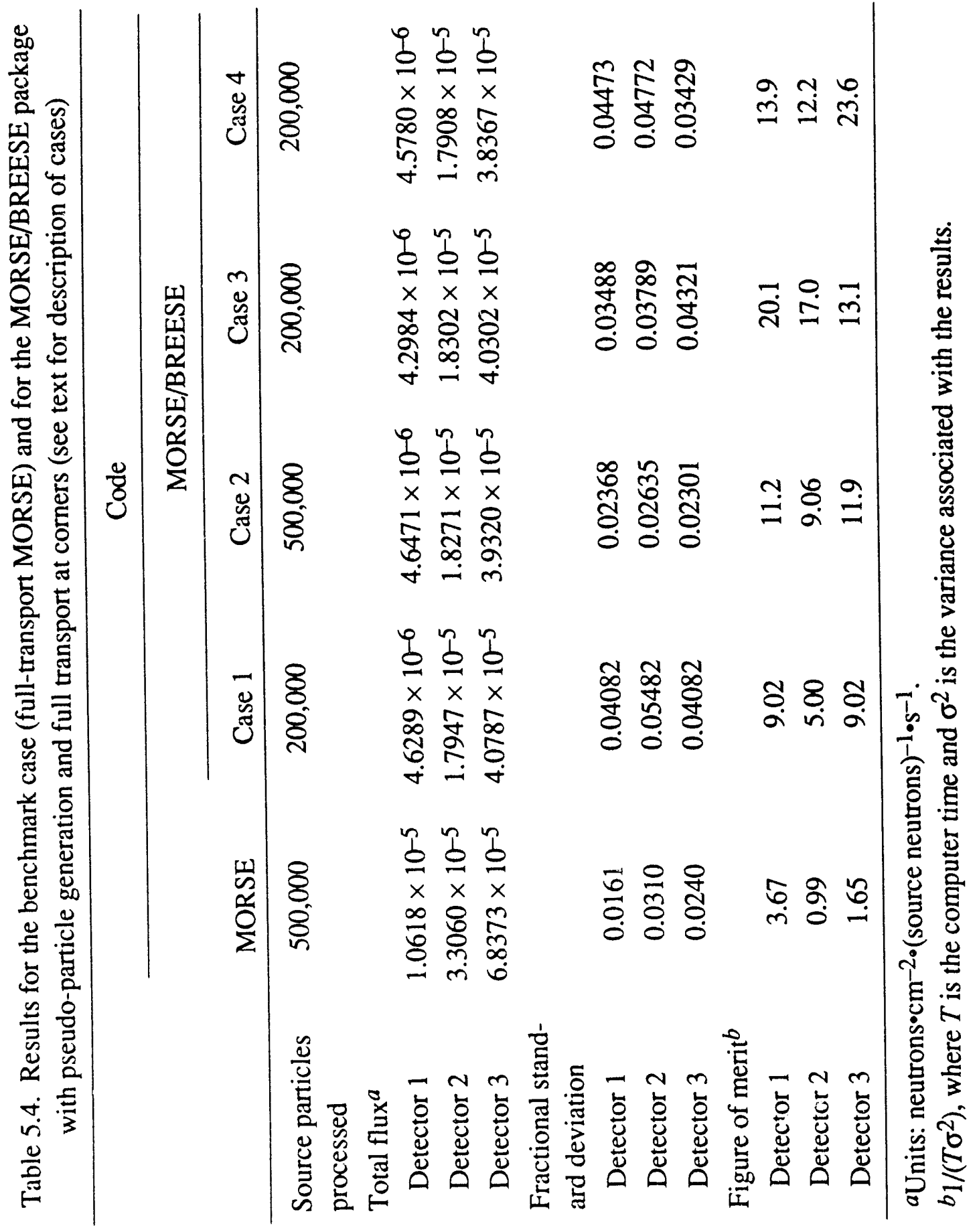


full transport at the corners. This difference can be explained by the position of the albedo surface behind the concrete wedge at the corner closest to the source. Pseudo-particles that would have been created at this surface would not be permitted to contribute to any detector; thus, no pseudo-particles were created at this surface. Results for detector 2 are only about $20 \%$ lower when a wedge of concrete is placed at the corners, as would be expected because of the lesser importance of the "lost" pseudo-particles with respect to the response of this detector. The improved performance of detector 3 is due to albedo particles scattered back from the corner albedo surface nearest the source (Fig. 5.2). This implies that pseudo-particles may represent the transport through corners very well and that placing a concrete wedge at the corners with an albedo surface behind the wedge has an adverse effect on the accuracy of the estimates.

\subsection{AVERAGE DISPLACEMENT OF THE POINT OF EMERGENCE}

As described in Sect. 4.2, the MCNP code was used to estimate the average displacement of the point of emergence from the point of incidence of an albedo event. Figures 5.3 and 5.4 show the radial distance and angular position distributions of the total albedo for a $14-\mathrm{MeV}$ neutron entering a concrete block

ORNL-DWG $91-3123$ FED

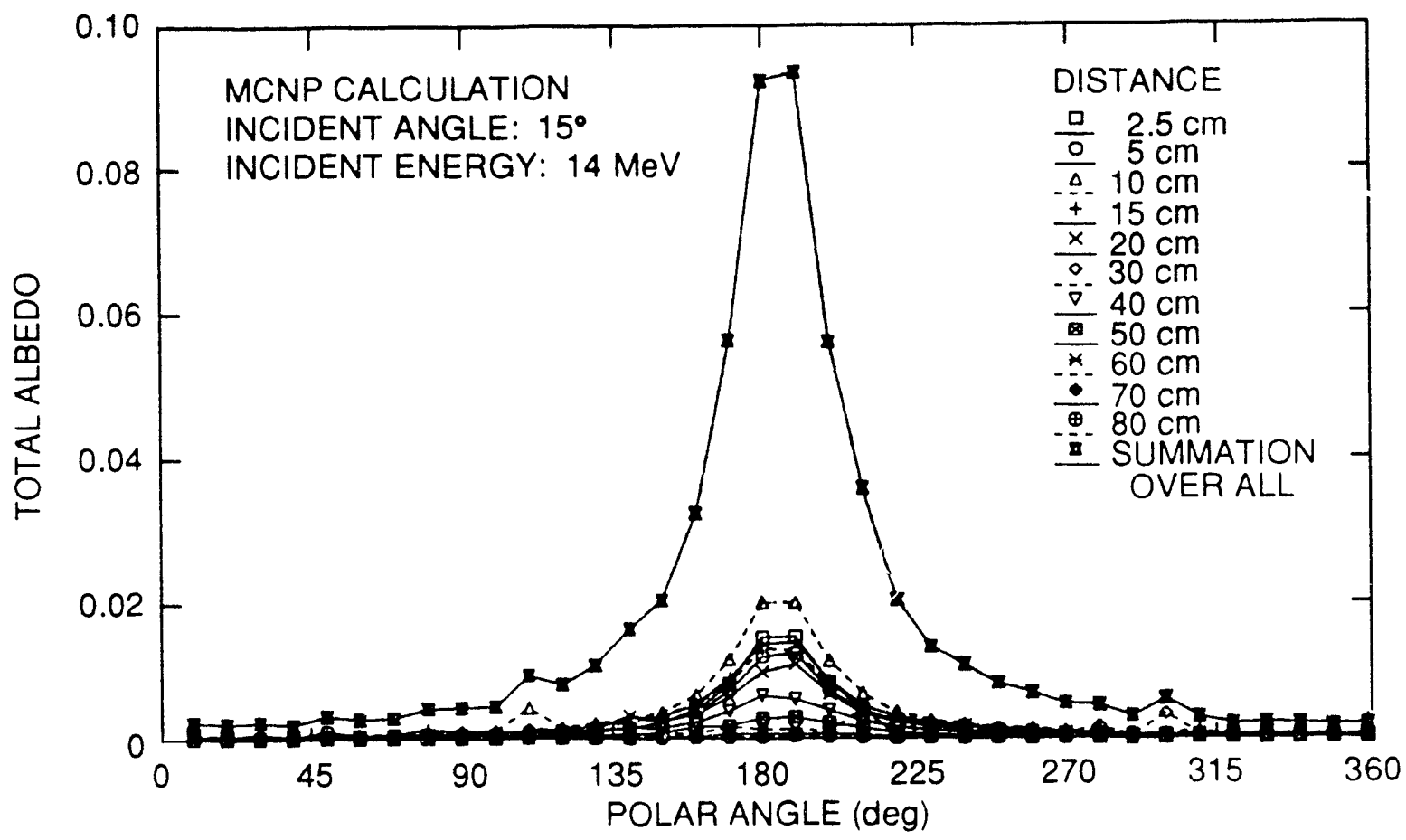

Fig. 5.3. Angular positions of emergent particles in relation to point of incidence. 


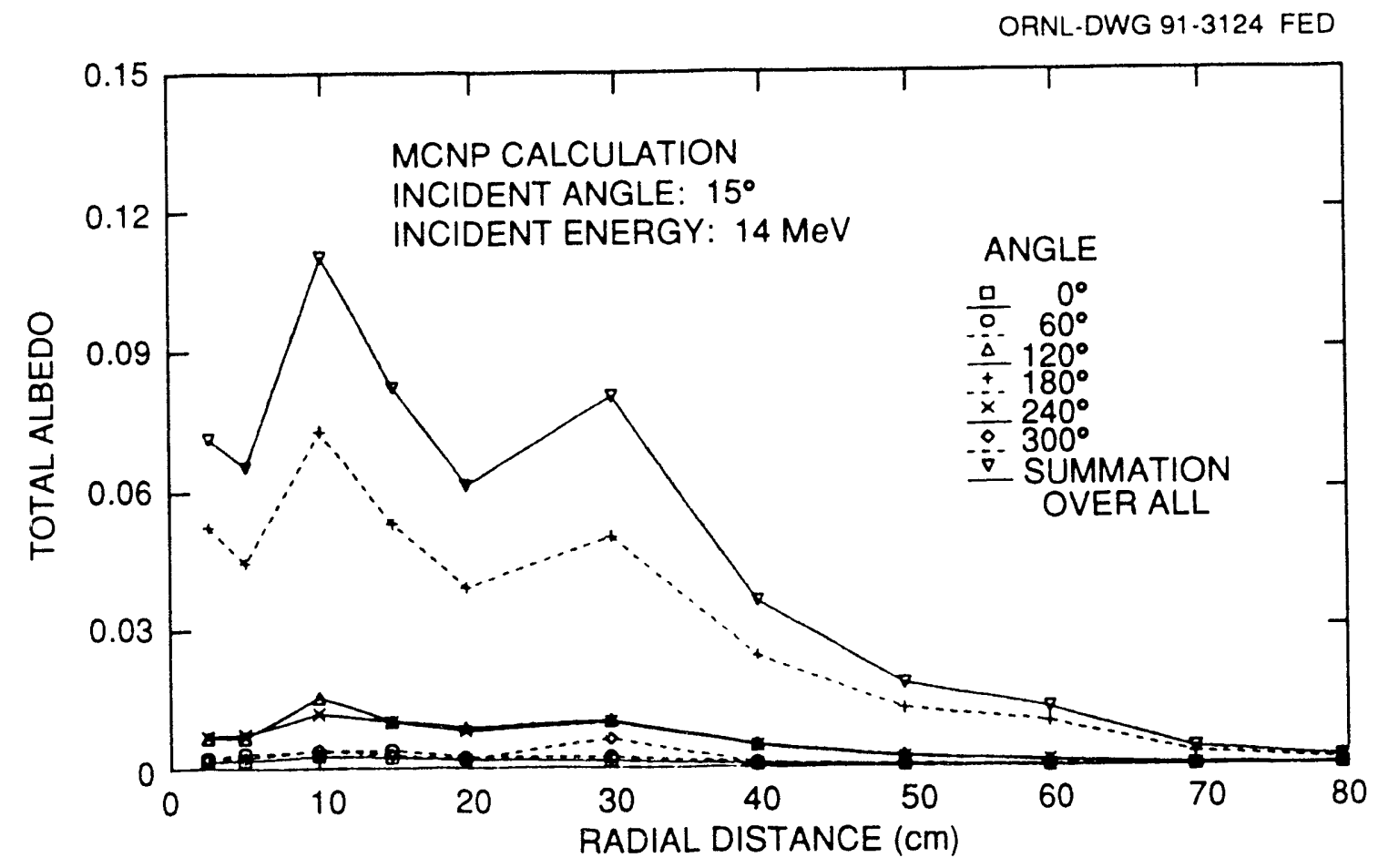

Fig. 5.4. Radial distance from point of incidence to point of emergence.

with a polar angle of $75^{\circ}$. The emergent directional distribution in Fig. 5.3 is highly peaked in the forward direction. Therefore, the point of emergence is more likely to be downstream from the point of incidence. Figure 5.4 shows that the displacement is significant and should not be neglected and that the most important component of the total displacement is that associated with the forward direction $\left(180^{\circ}\right)$.

Table 5.5 presents results obtained with the modified version of the MORSE/BREESE pachage that includes pseudo-particle generation and displacement of the point of emergence. Cases 1-4 represent different biasing techniques, as discussed in Sect. 5.2. The accuracy of the results (relative to the MORSE benchmark) improves from the previous $25 \%$ (low) for pseudo-particles without displacement to approximately $10 \%$ (low). This deviation remains constant for all three detectors, suggesting a hetter characterization of the radiation field, in contrast to the results presented in Tables 5.1-5.4.

Table 5.6 presents the results of calculations with pseudo-particle generation, displacement of the point of emergence, and full transport at the corners. Again, adding a concrete wedge decreases the estimated responses at detectors 1 and 2 . This consistency in the trends further demonstrates that pseudo-particles represent the particle transport through the corner better than the inclusion of a concrete wedge. The results with full transport at the corners are higher than those of 


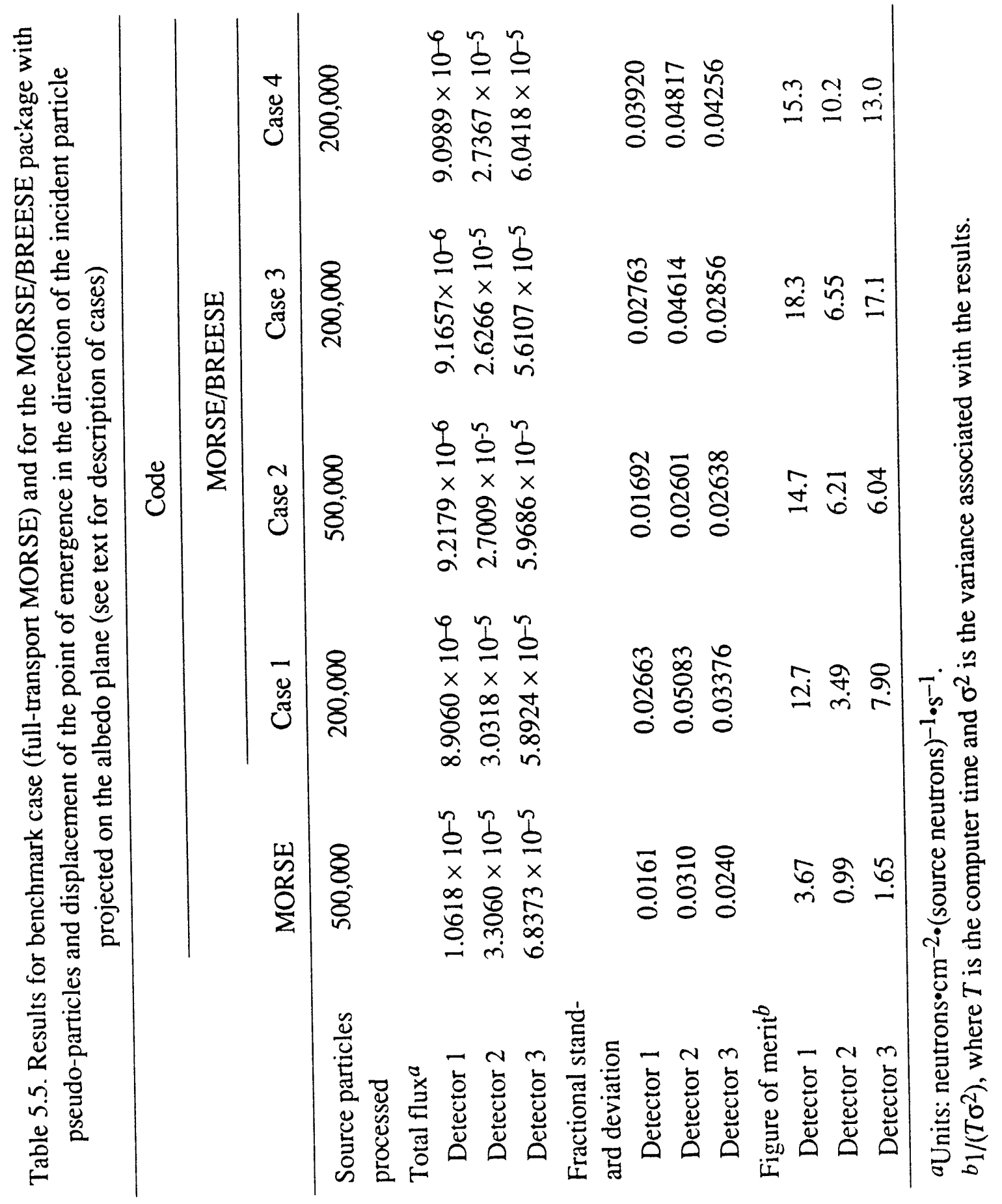




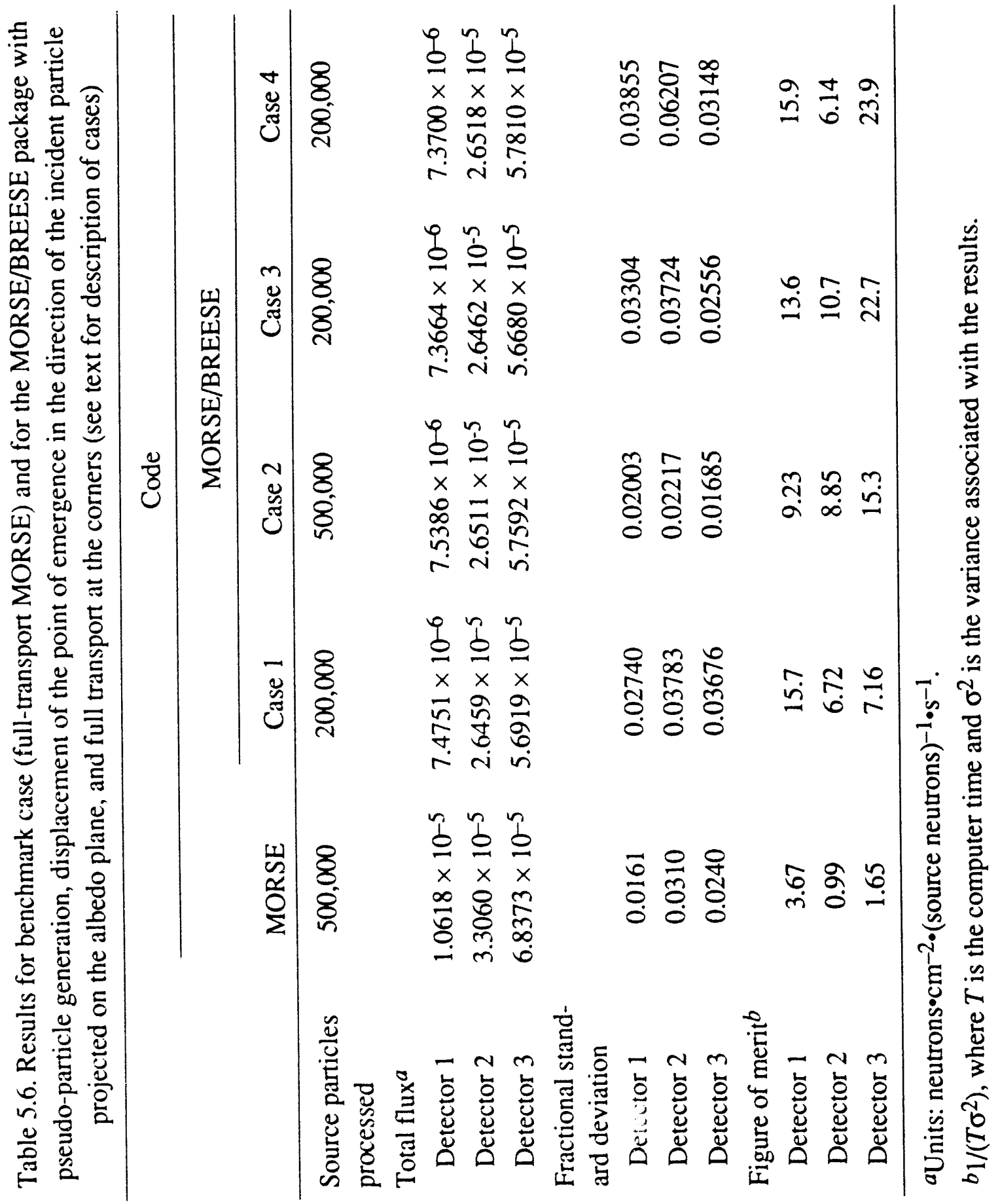


Table 5.4 because of the downstream displacement at the albedo surfaces behind the concrete wedges.

Another feature is the improvement by 20 to $30 \%$ in the figure of merit for the calculations that include the displacement of the point of emergence. This improvement is significant because it indicates that the additional computer time spent in performing the displacement phase of the albedo-transported particles was accompanied by a significant decrease in the variance, probably due to more uniform contributions to the detectors. In some cases, the figures of merit for results that included the displacement of the point of emergence decreased in value.

Table 5.7 presents results obtained by projecting the displacement vector (which has a magnitude equal to the absolute length of the displacement and a direction equal to that of the incident particle projected onto the albedo plane) onto the direction of the axis of the duct. Some improvement was obtained using this displacement procedure, in particular for the detector at the exit of the duct. In this procedure, no weight correction is applied to the albedo particle because of this "bias" in the direction of the displacement. The results indicate that this assumption is acceptable for the sample problem, but a universal application of this procedure may be questionable because of a tendency to underestimate the results at other positions (as can be seen by comparing the results presented in Tables 5.5 and 5.7).

Table 5.8 presents results for calculations with the displacement in the direction of the axis of the duct and full transport at the corners. These results are lower than those in Table 5.7 by about the same percentage that separates the results in Tables 5.5 and 5.6.

\subsection{GENERATION OF NEW ALBEDO DATA}

For the calculations discussed in Sects. 5.2 and 5.3, essentially all of the "lost" information for a particle experiencing an albedo event is recovered using the standard albedo data, but the results are still consistently low. Under these conditions, the validity of the standard albedo data must be questioned.

The assumption that the albedo data correctly describe the true reflection process was not supported by these calculations. A new set of calculations was performed with the MINP code to benchmark the angular distribution of the albedo data for a semi-infinite concrete medium in plane geometry. The polar angular distributions of the point albedos calculated with the MCNP code did agree with the distributions of the standard albedo data, but the azimuthal distributions of the available albedo data were shown to be more isotropic and the scattering more preferentially in the backward directions than indicated by MCNP distributions. Figures 5.5 and 5.6 show the azimuthal distributions calculated with the MCNP code and those from a standard albedo data set for two different combinations of incident polar angle and energy. The plots are displayed for five $\eta$ levels. Level 1 


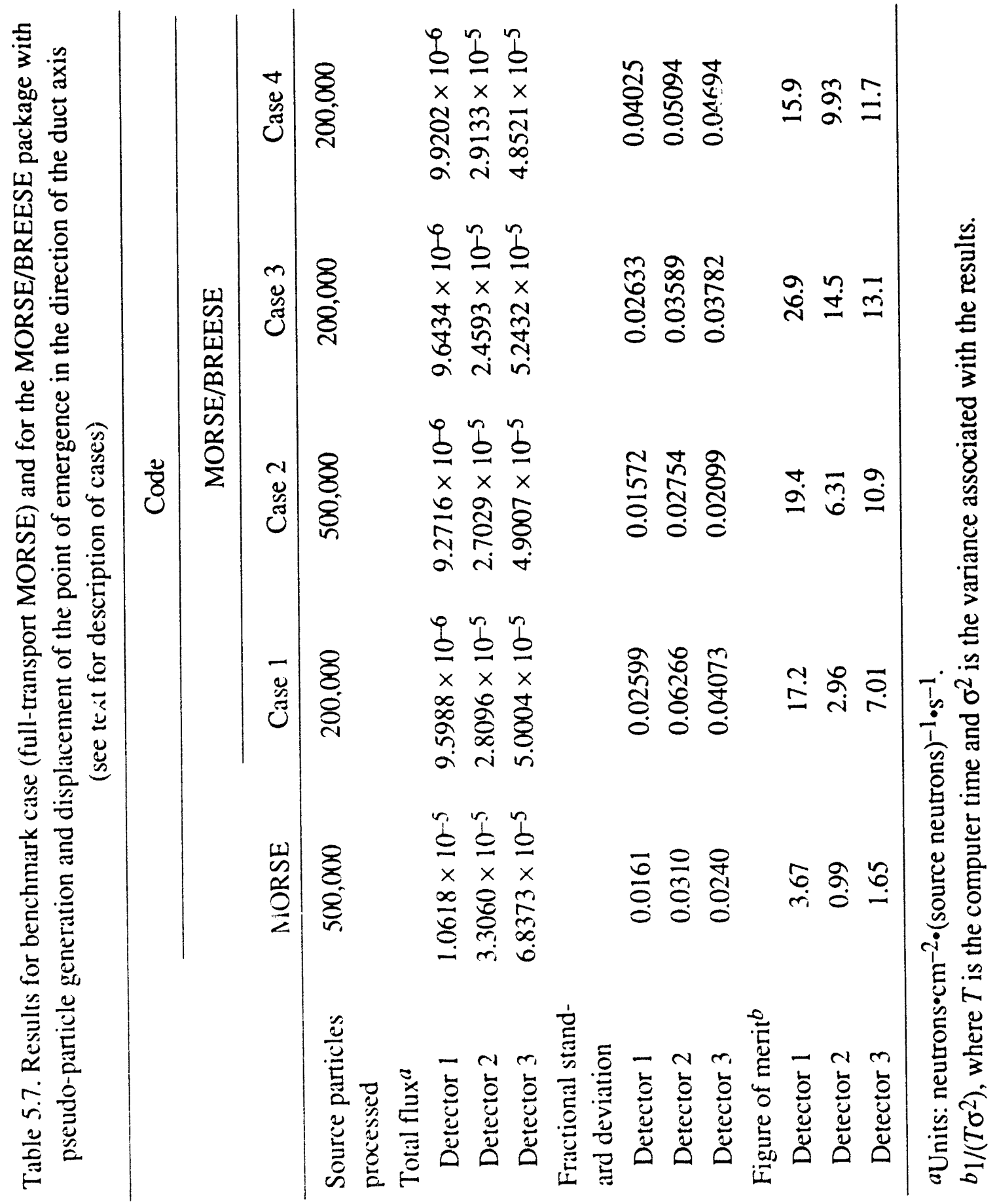




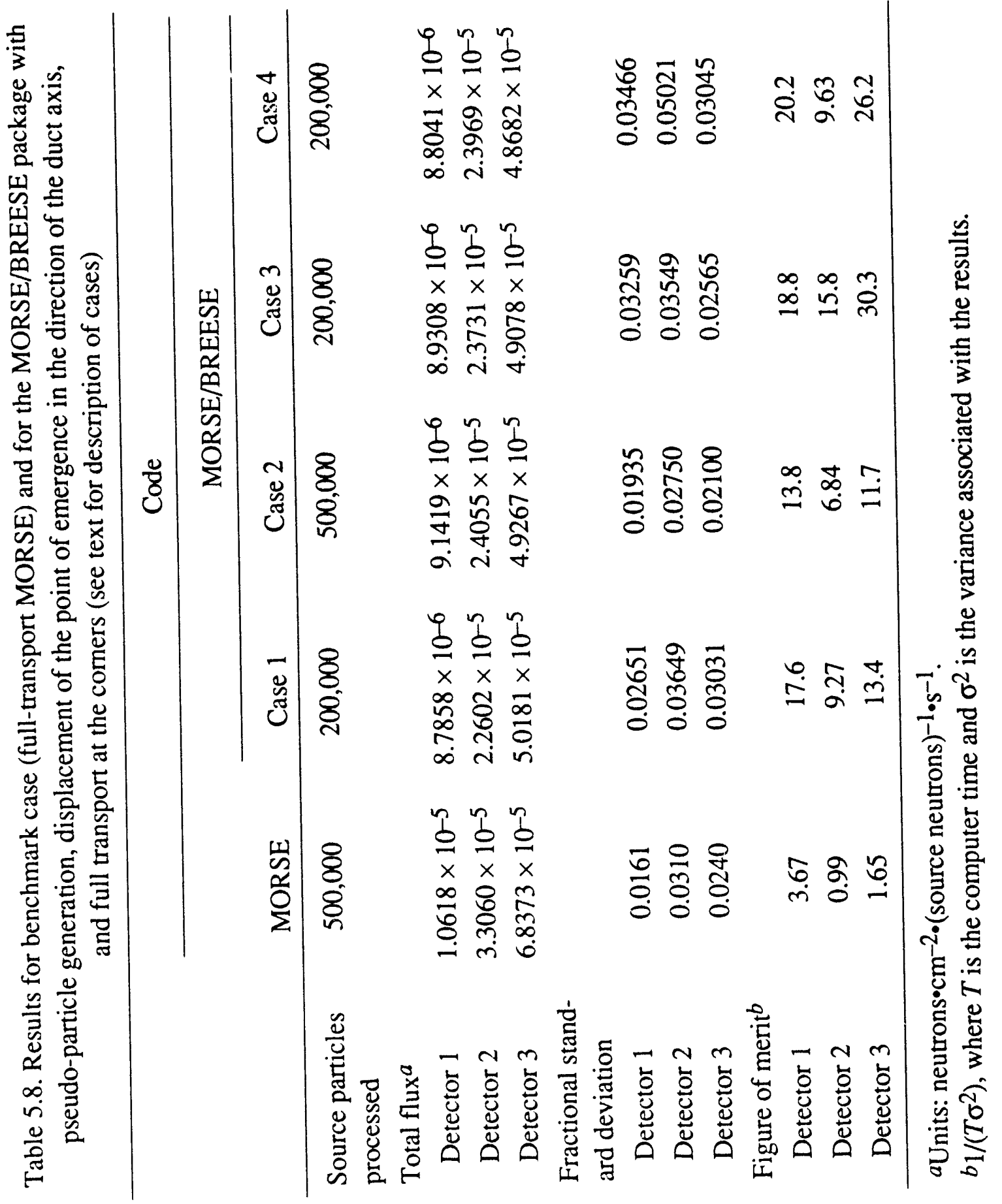


ORNL-DWG 91M-3125 FED

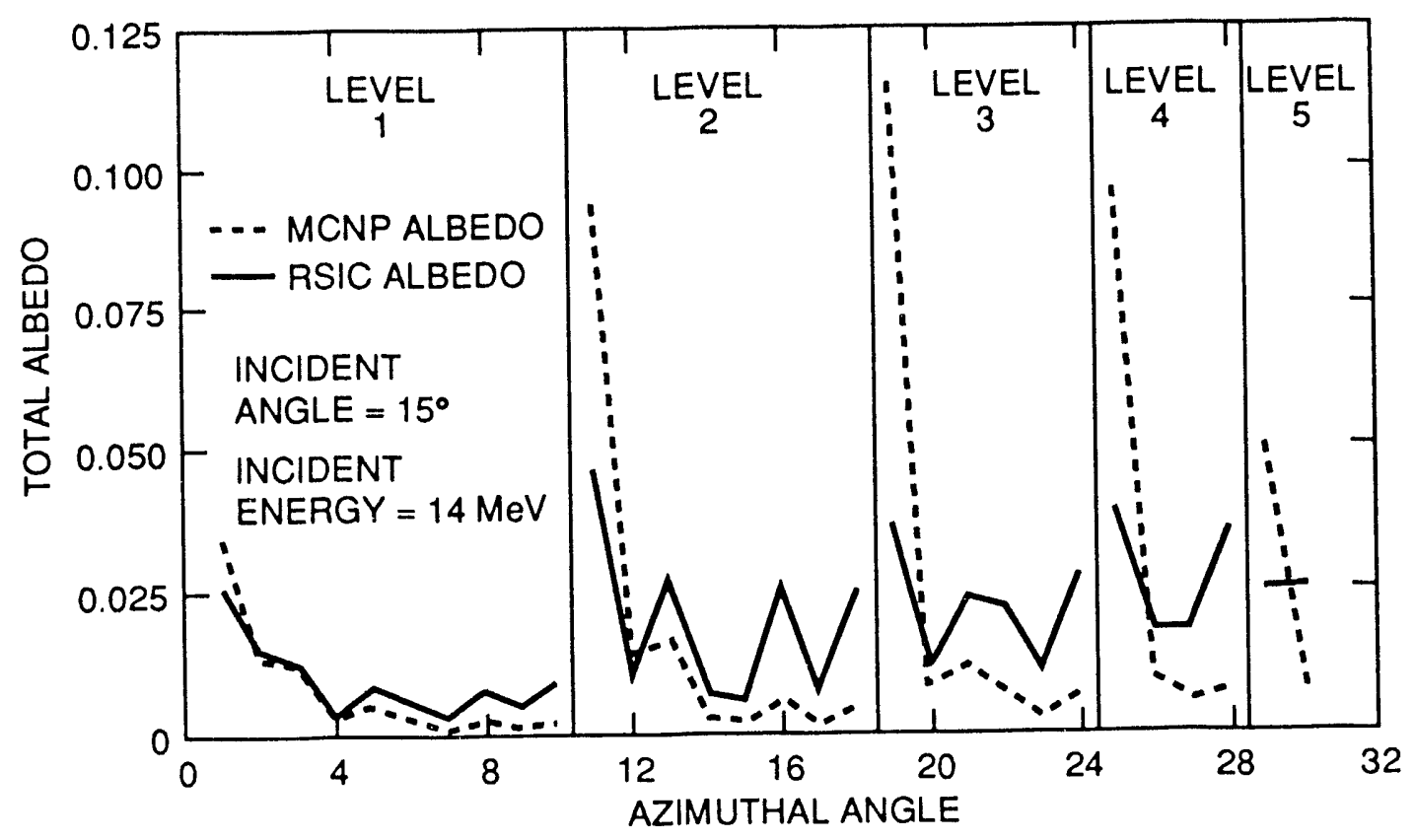

Fig. 5.5. Angular azimuthal distribution of the available albedo data and of the MCNP calculated data for an incident polar angle of $75^{\circ}$ and a neutron energy of $14 \mathrm{MeV}$.

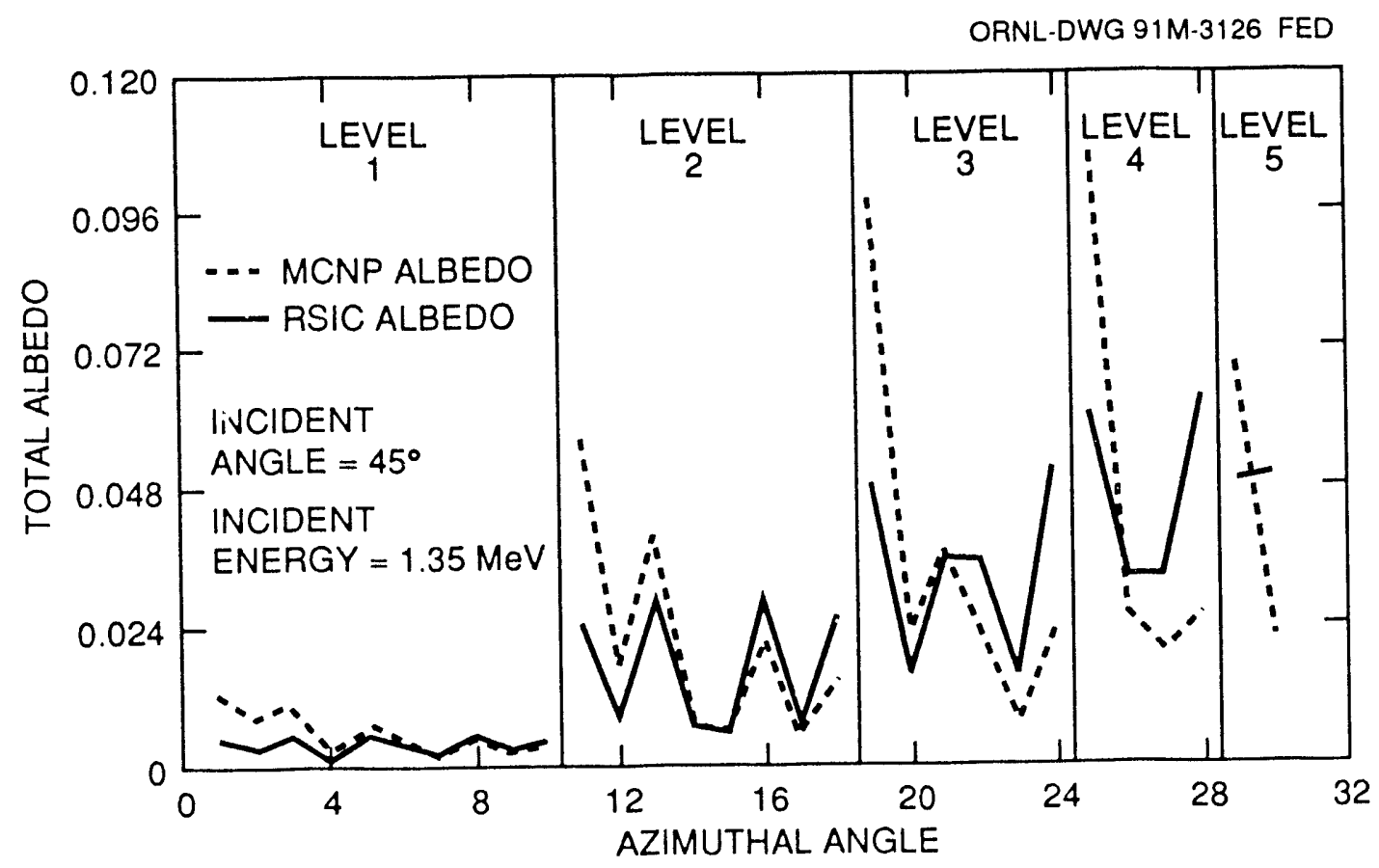

Fig. 5.6. Angular azimuthal distribution of the CARP albedo data (ref. 7) and of the MCNP albedo information for an incident polar angle of $45^{\circ}$ and a neutron energy of $1.35 \mathrm{MeV}$. 
corresponds to the largest and level 5 to the smallest cosine of the polar angle in the quadrature set. Within each level, smaller abscissa values correspond to more forward azimuthal angles.

To better understand the poor correlation of the available albedo data with the MCNP-estimated azimuthal distributions, the angular flux distributions generated by the discrete ordinates code DOT, which has been the main tool for the calculation of albedo data, were analyzed. It was found that negative fluxes were calculated for many discrete directions and that the negative fluxes occurred more frequently for high neutron energy groups. These negative fluxes are usually associated with the forward directions, and they are at least in part due to the low-order expansion of the cross sections. In the BREESE code, negative fluxes are set equal to zero, thereby reducing the magnitudes of the forward-directed components. The values of the total albedo given by the CARP code are not consistent with those produced by the BREESE code. After the negative fluxes are set equal to zero by the BREESE code, the value for the total albedo more than doubles for several combinations of incident energy group and direction. In addition, in the sampling scheme implemented in the CARP/BREESE system, point albedos are characterized in terms of three phase-space coordinates of the emergent particle-energy, $\eta$ level, and azimuthal angle. In the BREESE code, an $\eta$ level is selected from the marginal probability distribution for the polar angle, then an azimuthal angle is selected from the conditional probability distribution for the azimuthal angle, and finally the energy is selected from the doubly conditional energy distribution. The problem for concrete is that the thermal neutron component is very large because of the presence of hydrogen, and the angular distribution, which is independent of the outgoing energy in the sampling scheme used in BREESE, is almost completely dominated by the nearly isotropic angular distribution of the thermal neutrons. This explains in part the isotropic character of the angular distribution of the DOT-generated albedo data, and because of the negative fluxes in the basic data, these distortions cannot be corrected.

On the basis of these results, a decision was made to generate a new set of albedo data. The usual discrete ordinates procedures for generating albedo data can calculate only point albedos, and Monte Carlo codes require prohibitively large amounts of computer time to obtain detailed albedo information with acceptable precision. A 3-D discrete ordinates code can provide a detailed description of the radiation field while requiring an acceptable amount of computer time. The $3-\mathrm{D}$ discrete ordinates code TORT was used to generate a new set of albedo data, as described in Sect. 4.3. Problems related to negative angular fluxes were addressed, and input options that provide positive fluxes for all combinations of mesh spaces, quadrature directions, and energy groups were selected.

Figure 5.7 displays the angular distribution of the CARP-generated point albedo; the MCNP-calculated albedo information, with the spatial variable collapsed to a point; and the TORT-calculated albedo data with $P_{3}$ and $P_{8}$ cross 
ORNL-DWG 91M-3127 FED

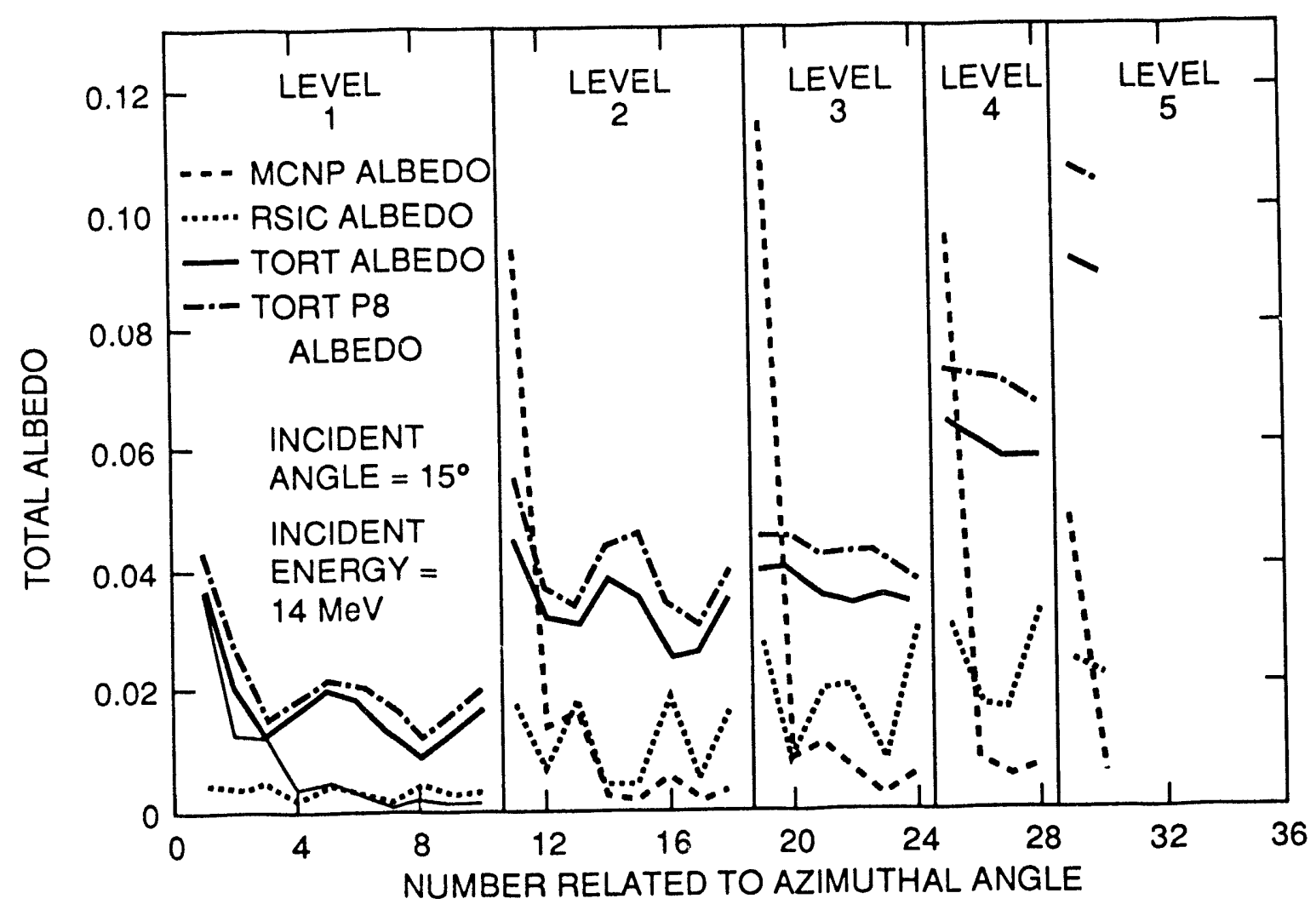

Fig. 5.7. Angular distributions of various albedo data sets.

sections, which also have the spatial variable collapsed to a point for display as point albedos. The order numbers displayed on the $x$-axis of Fig. 5.7 are related to the azimuthal angle in such a way that smaller numbers within each level correspond to more negative cosines of the azimuthal angle. Note that the quadrature set used for generating TORT albedo data is different from that used for generating CARP and MCNP albedos. Table 5.9 presents the limits of the polar angles for the two quadrature sets used. The level numbers are the same as those in Figs. 5.5-5.7. The limits of the azimuthal angle within each $\eta$ level for each quadrature set are presented in Table 5.10.

Table 5.9. Limits of the polar angles of the CARP and MCNP albedo data and of the polar angles of the TORT albedo data

\begin{tabular}{ccc|c|c|c|c|}
\hline & \multicolumn{7}{c|}{$\cos \Theta$ limit } \\
\cline { 2 - 8 } & Level 5 & Level 4 & Level 3 & Level 2 & Level 1 \\
\cline { 2 - 8 } CARP/MCNP & 1.0 & 0.93333 & 0.78388 & 0.56479 & 0.29552 & 0 \\
TORT & 1.0 & 0.90800 & 0.76825 & 0.61728 & 0.37313 & 0 \\
\hline
\end{tabular}




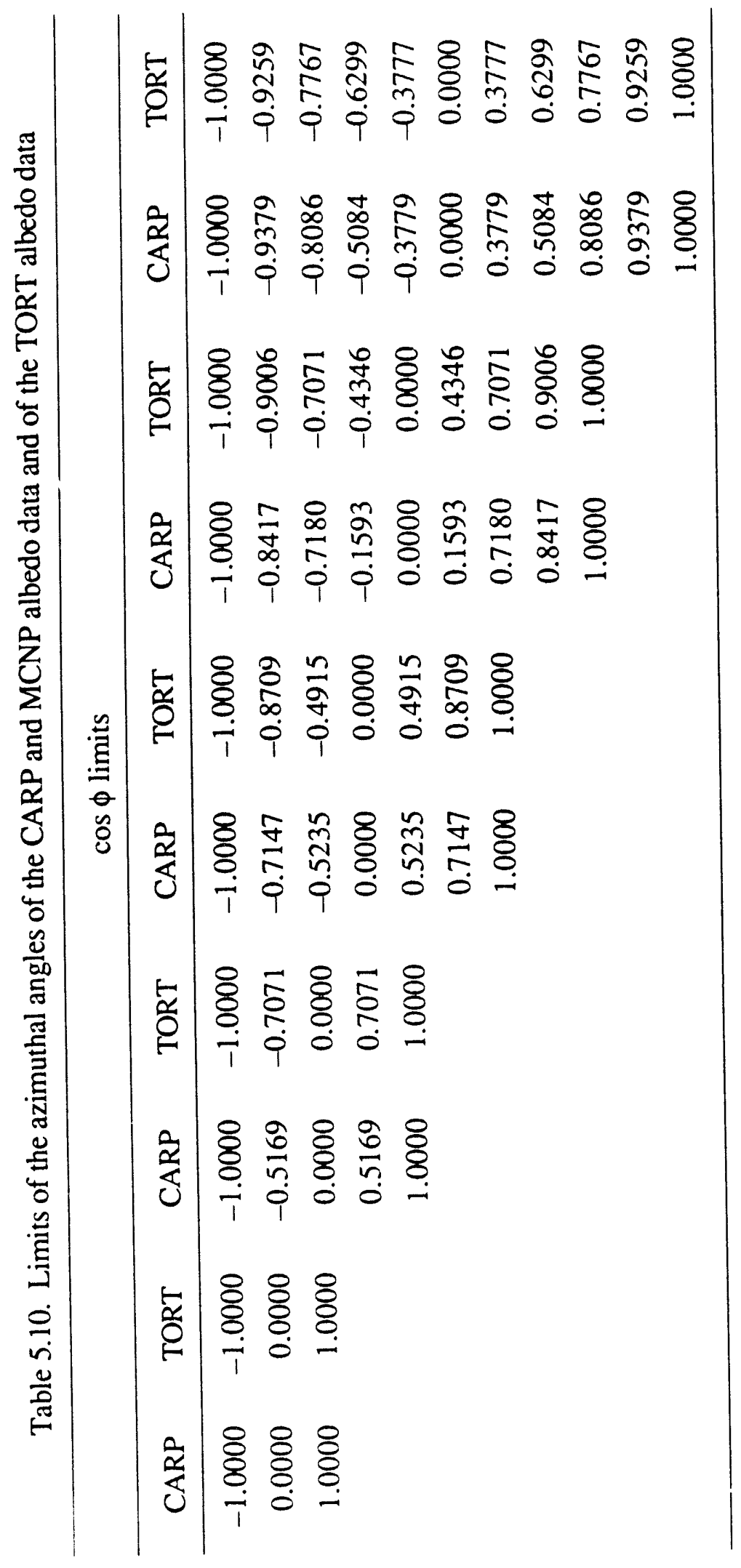


Figure 5.8 displays the spatial distributions of the TORT albedos with $\mathrm{P}_{3}$ cross sections.

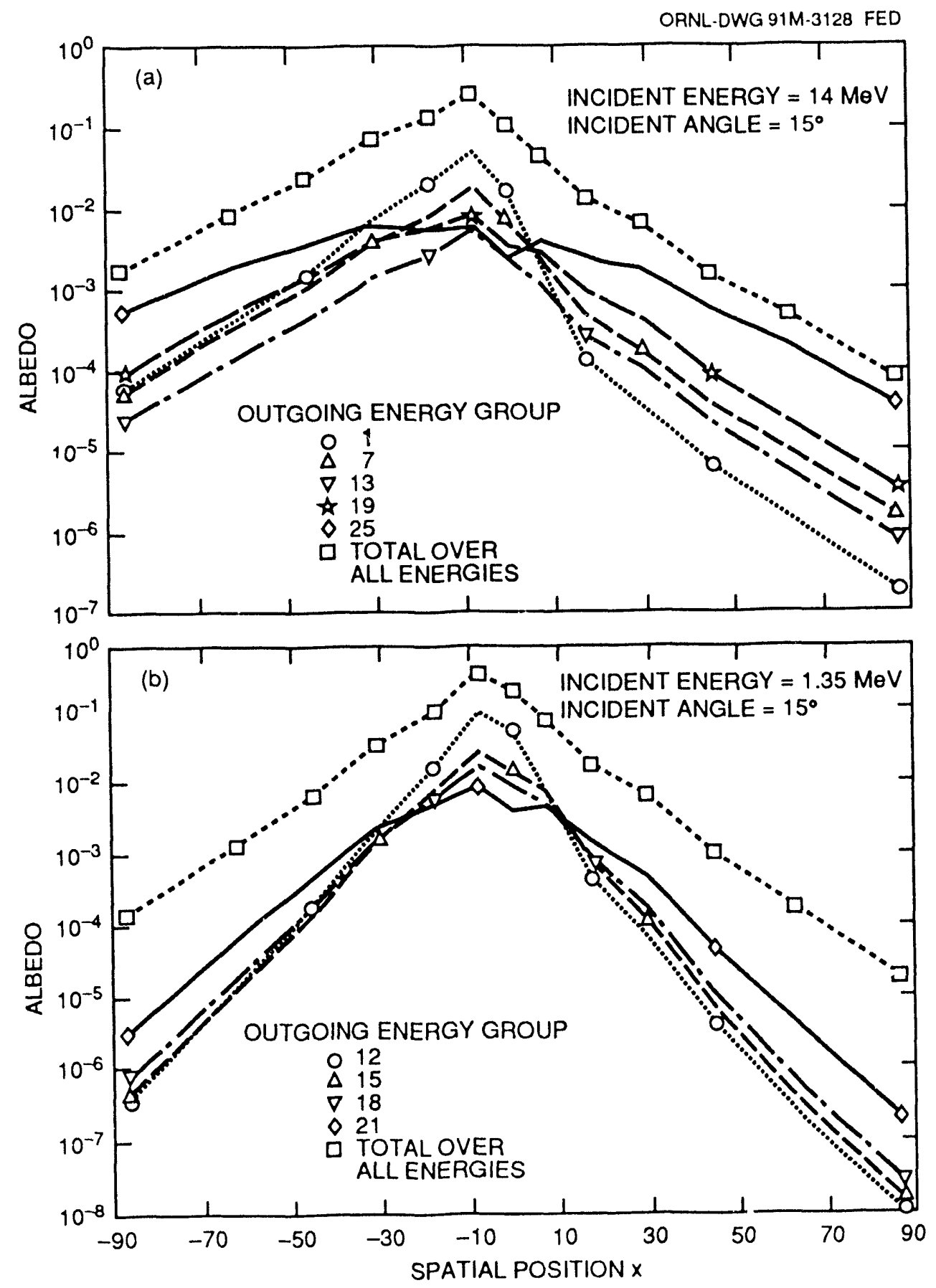

Fig. 5.8. Spatial distributions of the TORT albedos for (a) an incident polar angle of $75^{\circ}$ and an incident energy of $14 \mathrm{MeV}$ and (b) an incident polar angle of $75^{\circ}$ and an incident energy of $1.35 \mathrm{MeV}$. 


\subsection{SPATIALLY DEPENDENT ALBEDOS}

\subsubsection{Forward Mode}

As described in Sect. 4.3.2, the MORSE code was modified to accommodate the 3-D albedo data. The ALBFAC3D interface code was written to format the albedo information generated by TORT into a file that can be read by the MORSE/STORM code package. Table 5.11 presents results obtained with the MORSE/STORM code using spatially dependent albedos for the sample problem of Sect. 5.1.

The Monte Carlo estimates of the effects of interest for detectors 1 and 2 using 3-D albedos are essentially the same as those of the MORSE benchmark case. The resuits for detector 3 are sligintly higher than for the benchmark case. However, they agree well with the results presented in Table 5.1 for the MCNP benchmark case. Only $17 \%$ of the total contributions at detector 1 come from albedo events; the remaining $83 \%$ come from real collisions of the pseudo-particles. This breakdown of the two components of the response explains the agreement of the MORSE benchmark response and the MORSE/STORM response for this detector. The next-event statistical estimates and the scattering of the pseudoparticles are performed using the standard procedures implemented in the MORSE code, and, as would be expected, the MORSE/STORM results are closer to the MORSE benchmark results than to the MCNP benchmark results. The difference between the MORSE and MCNP senchmarks can be explained by the cross section treatments and by differences in scattering and estimation procedures. The MORSE benchmark calculation was performed using $P_{3}$ cross sections and the standard scattering procedure; the scattering process is described by two outgoing angles. This procedure does not affect the results after a few collisions, but becanse of the small size of the sample problem the first-and second-collided components of the radiation field are dominant. This leads to the conclusion, for this case, that some of the differences between the MORSE and MCNP benchmark results are due to the MORSE treatment of the scattering event. The next-event contributions to the effect of interest are also affected by the low order of truncation of the Legendre polynomials. For this particular problem, which is due to a highly localized region of importance for each detector, the $\mathrm{P}_{3}$ representation of the scattering process may not be sufficiently accurate.

Detector 3, which is aligned with the source through the axis of the first leg of the penetration, receives $22 \%$ of its contributions from albedo events and $8 \%$ from the uncollided component. The fact that the results for this detector are closer to the MCNP benchmark suggests that for this particular detector ray-like effects were present in the MORSE full-transport case. 


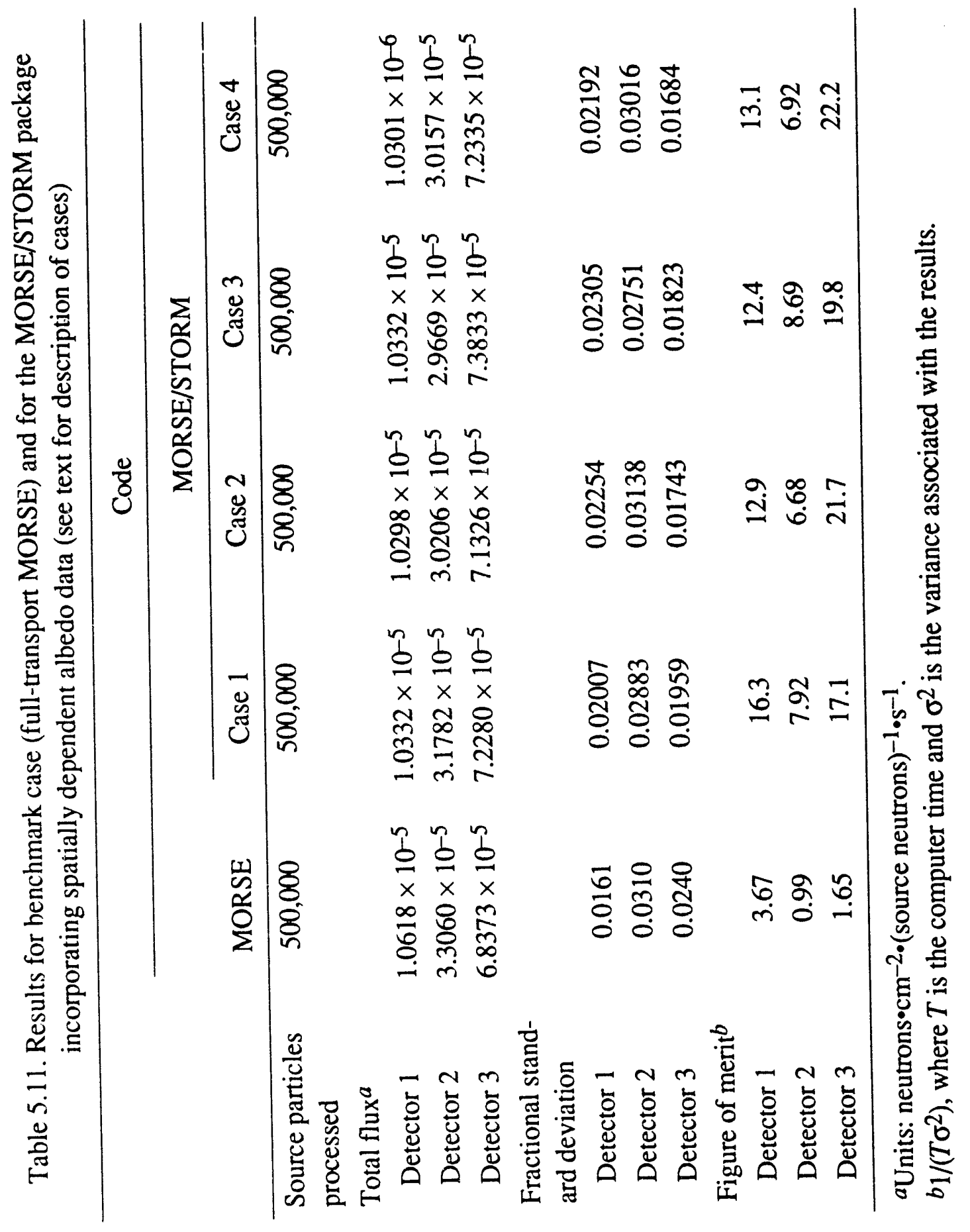




\subsubsection{Adjoint Mode}

The MORSE code can perform both forward and adjoint analysis. The adjuncton albedos can be obtained from the same forward angular fluxes used in generating the forward albedos. The ALBFAC3D interface code can process the forward angular flux file generated by the TORT code and synthesize either forward or adjuncton albedo data. The MORSE/STORM code can also read the albedo data file and perform calculations in either the forward mode or the adjoint mode. Table 5.12 presents the results of calculations with adjuncton albedos and with the standard adjoint mode of analysis in the MORSE code. The geometric configurations used in these calculations are the same as those used in the forward calculations. Only the three top energy groups were used for the adjoint calculation. The adjoint source is located at the position of detector 1 (Fig. 5.2), and the adjoint estimate is performed to an adjoint point detector located at the center of the entrance of the penetration-this is an approximation, since the source in the forward calculation is uniformly distributed over the entrance. The energy spectrum of the forward source (adjoint response function) is the same as that of the forward calculations (14-MeV neutron source). The results of Table 5.12 indicate that the adjoint calculation performed using adjuncton albedos yields statistically the same result as the full-transport calculation and that the figure of merit is $50 \%$ larger when adjuncton albedos are used. Both calculations were performed without biasing techniques, which shows that the use of adjuncton albedos improves the computational efficiency.

Table 5.13 compares the MORSE/STORM adjuncton albedo calculation for the standard (complete) sample problem with MORSE and MCNP benchmarks. For the MORSE and MCNP benchmarks, the standard forward mode calculations

Table 5.12. Results of full-transport MORSE code adjoint mode calculation, performing full transport of adjunctons without biasing, and of standard MORSE/STORM code adjoint mode calculation, using adjuncton albedos without biasing of pseudo-adjuncton particles, for the three-group sample problem at detector 1

\begin{tabular}{|c|c|c|}
\hline & \multicolumn{2}{|c|}{ Code } \\
\hline & $\begin{array}{l}\text { Full-transport } \\
\text { MORSE }\end{array}$ & $\begin{array}{l}\text { Adjuncton albedo } \\
\text { MORSE/STORM }\end{array}$ \\
\hline Source particles processed & 600,000 & 500,00 \\
\hline Total flux ${ }^{a}$ & $2.20371 \times 10^{-6}$ & $2.26881 \times 10^{-6}$ \\
\hline Fractional standard deviation & 0.02622 & 0.02315 \\
\hline Figure of merit $b$ & 32.1 & 48.3 \\
\hline
\end{tabular}

aUnits: neutrons $\cdot \mathrm{cm}^{-2} \cdot(\text { source neutrons })^{-1} \cdot \mathrm{s}^{-1}$.

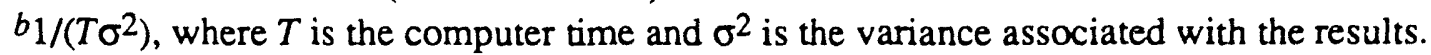


Table 5.13. Results for benchmark cases (full-transport MORSE and MCNP) and for the MORSE/STORM adjuncton-albedo calculation for the three-group sample problem at detector 1

\begin{tabular}{|c|c|c|c|}
\hline & \multicolumn{3}{|c|}{ Code } \\
\hline & MORSE & MCNP & $\begin{array}{c}\text { Adjoint } \\
\text { MORSE/STORM }\end{array}$ \\
\hline $\begin{array}{l}\text { Source particles } \\
\text { processed }\end{array}$ & 500,000 & $3,000,000$ & $4,200,000$ \\
\hline Total flux ${ }^{a}$ & $1.0618 \times 10^{-5}$ & $1.15532 \times 10^{-5}$ & $1.23179 \times 10^{-5}$ \\
\hline $\begin{array}{l}\text { Fractional standard } \\
\text { deviation }\end{array}$ & 0.0161 & 0.0079 & 0.0635 \\
\hline Figure of merit $b$ & 3.67 & 24.0 & 3.74 \\
\hline
\end{tabular}

a Units: neutrons $\cdot \mathrm{cm}^{-2} \cdot(\text { source neutrons) })^{-1} \cdot \mathrm{s}^{-1}$.

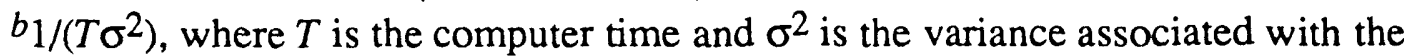
results.

with full transport of particles and no biasing were performed. This calculation is a severe test of the adjuncton albedo calculation because the adjoint response, a $14-\mathrm{MeV}$ point neutron source, creates an inherently difficult problem to solve in the adjoint mode. Also, the decision not to use biasing procedures when performing any of the Monte Carlo random walks precluded the optimization of the adjoint calculation, which for the sample problem would involve energy biasing.

\section{CONCLUSIONS AND FUTURE WORK}

This research demonstrated new procedures that permit more generalized use of the albedo methodology in both forward and adjoint modes of Monte Carlo calculation. With the use of "pseudo-particles," it was possible to recover an essential part of the information that would have been lost at an albedo event. This recovered information was used to calculate a missing and very important component in the statistical estimation procedure. The spatial variable was included in the albedo procedure either through Monte Carlo-generated supplementary information or through directly calculated, spatially dependent albedo data. It was also demonstrated that, owing to the limitations of point albedos in accurately representing the angular distribution of the reflection process, the modified version of the MORSE/BREESE code pacliage that includes both the displacement of the point of emergence of an albedo event and the tracking of pseudo-particles does not yield the same results as the benchnark calculations. The MORSE/STORM code 
package, which was developed to process and use spatially dependent albedo data calculated by the 3-D discrete ordinates code TORT, demonstrated close agreement with the benchmark results to within the statistical uncertainties.

An important conclusion from this rescarch is that the spatially dependent albedo data associated with the generation of pseudo-particles can be used in any class of problems. It was demonstrated that all significant components of the radiation field can be correctly represented by a MORSE/STORM calculation when spatially dependent albedos are used.

The sample problem was carefully designed to make it a valid test for certifying the new albedo procedures. The Monte Carlo estimate of the effect of interest is strongly influenced by several components of the radiation field. The transmission of particles through the material medium is on balance with the streaming through the duct. The smal! size of the problem allows a large fraction of particles to escape from the system after a few collisions. The fact that the problem does not present a single dominant component of the radiation field ensures that traditional albedo Monte Carlo techniques would not yield satisfactory results. The procedures implemented in the MORSE/STORM code, for both forward and adjoint modes, were demonstrated to efficiently characterize the radiation field. The results of both forward and adjoint calculations agree with the benchmark results. The figures of merit for both modes show considerable improvement over those for full-transport Monte Carlo calculations.

The biasing procedures implemented in the MORSE/STORM code have satisfactorily controlled the number of pseudo-particles created in different parts of the geometry. The optimal use of these procedures may greatly improve the Monte Carlo figure of merit for calculations in which the importance function is strongly dependent on the phase-space coordinates.

Standard biasing procedures were not used during the tracking of either albedo particles or pseudo-particles. The implementation of biasing procedures with respect to the spatially dependent albedo data is an almost straightforward task that may lead to important improvements in the figure of merit. For example, biasing the displacement of albedo particles can reduce the number of pseudoparticles created due to displacement into regions of low importance. A concept similar to the "nonleakage" biasing procedure can be used to keep the displaced albedo particles inside a duct when solving some classes of problems in which the only important component is the streaming component.

The generation of spatially dependent albedo data was performed using a standard production version of the TORT code, and many improvements should be possible. Modifying the "sweeping of the spatial mesh" procedure to take advantage of the spatial symmetry of the albedo generation calculation is one of several possible improvements that deserve attention. Another exercise that would reduce the computer time required to generate $3-\mathrm{D}$ albedo data would be a st udy of the sensitivity of the accuracy of the generated albedo information to the modeling of the spatial mesh. Also, the angular and spatial distributions of the albedo data can benefit from an optimization of the number and/or the size of the spatial mesh. 


\section{REFERENCES}

1. M. B. Emmett, The MORSE Monte Carlo Radiation Transport Code System, ORNL-4972/R1, Union Carbide Corp., Nuclear Div., Oak Ridge National Laboratory, February 1983.

2. J. F. Briesmeister, T. E. Booth, and D. G. Collins, MCNP: A General Monte Carlo Code for Neutron and Photon Transport, Version 3A, LA-7396-M, Rev. 2, Los Alamos National Laboratory, Los Alamos, N.M., 1986.

3. V. R. Cain and M. B. Emmett, BREESE-II: Auxiliary Routines for Implementing the Albedo Option in the MORSE Monte Carlo Code, ORNL/TM6807, Union Carbide Corp., Nuclear Div., Oak Ridge National Laboratory, July 1979.

4. F. R. Mynatt et al., The DOT III Two-Dimensional Discrete Ordinates Transport Code, ORNL/TM-4280, Union Carbide Corp., Nuclear Div., Oak Ridge National Laboratory, 1973.

5. W. A. Rhoades and R. L. Childs, The TORT Three-Dimensional Discrete Ordinates Neutron/Photon Transport Code, ORNL-6268, Martin Marietta Energy Systems, Inc., Oak Ridge National Laboratory, November 1987.

6. I. C. Gomes, A Generalized Albedo Option For Forward And Adjoint Monte Carlo Calculations, Ph.D. dissertation, the University of Tennessee, Knoxville, 1991.

7. M. B. Emmett and W. A. Rhoades, CARP, A Compuier Code and Albedo Data Library for Use by BREESE, the MORSE Albedo Package, ORNL/TM-6503, Union Carbide Corp., Nuclear Div., Oak Ridge National Laboratory, 1978. 
ORNL/FEDC-91/1

Dist. Category UC-423, 424

\section{INTERNAL DISTRIBUTION}

1. Director, ORNL Fusion Energy Division

2. C. C. Baker

3. M. J. Saltmarsh

4. L. A Berry

5. B. A. Carreras

6. R. A. Dory

7. J. L. Dunlap

8. H. H. Haselton

9. M. S. Lubell

10. T. E. Shannon

11. R. P. Leinius
12-13. Laboratory Records Department

14. Laboratory Records, ORNL-RC

15-16. Central Research Library

17. Document Reference Section

18. Fusion Energy Division Library

19-20. Engineering Technology/Fusion Energy Division Publications Office

21. ORNL Patent Office

22-26. I. C. Gomes

27-30. P. N. Stevens

31. M. B. Emmett

\section{EXTERNAL DISTRIBUTION}

32. Office of the Assistant Manager for Energy Research and Development, U.S. Department of Energy Field Office, Oak Ridge, P.O. Box 2000, Oak Ridge, TN 37831

33. N. A. Davies, Director, Office of Fusion Energy, Office of Energy Research, ER50 Germantown, U.S. Department of Energy, Washington, DC 20545

34. M. Roberts, International Programs, Office of Fusion Energy, Office of Energy Research, ER-52 Germantown, U.S. Department of Energy, Washington, DC 20545

35. D. E. Baldwin, Institute for Fusion Studies, The University of Texas at Austin, RLM 11.222, Austin, TX 78712-1060

36. R. W. Conn, Mechanical, Aerospace, and Nuclear Engineering Department, 6291 Boelter Hall, University of California, Los Angeles, CA 90024-1597

37. R. Parker, Plasma Fusion Center, Massachusetts Institute of Technology, 167 Albany St., NW16-288, Cambridge, MA 02139

38. K. I. Thomassen, L-637, Lawrence Livermore National Laboratory, P.O. Box 5511, Livermore, CA 94550

39. J. D. Callen, Department of Nuclear Engineering, University of Wisconsin, Madison, WI 53706-1687

40. S. O. Dean, Fusion Power Associates, Inc., 2 Professional Drive, Suite 248, Gaithersburg, MD 20879

41. H. K. Forsen, Bechtel Group, Inc., Research Engineering, P.O. Box 3965, San Francisco, CA 94119

42. R. W. Gould, Department of Applied Physics, California Institute of Technology, Pasadena, CA 91125 
43. R. A. Gross, Plasma Research Laboratory, Columbia University, New York, NY 10027

44. R. J. Hawryluk, Princeton Plasma Physics Laboratory, P.O. Box 451, Princeton, NJ 08543

45. D. M. Meade, Princeton Plasma Physics Laboratory, P.O. Box 451, Princeton, NJ 08543

46. W. M. Stacey, School of Nuclear Engineering and Health Physics, Georgia Institute of Technology, Atlanta, GA 30332

47. D. Steiner, Nuclear Engineering Department, NES Building, Tibbetts Avenue, Rensselaer Polytechnic Institute, Troy, NY 12181

48. R. Varma, Physical Research Laboratory, Navrangpura, Ahmedabad 380009, India

49. Bibliothek, Max-Planck Institut für Plasmaphysik, Boltzmannstrasse 2, D-8046 Garching, Federal Republic of Germany

50. Bibliothek, Institut für Plasmaphysik, KFA Jülich GmbH, Postfach 1913, D-5170 Jülich, Federal Republic of Germany

51. Bibliothek, KfK Karlsruhe GmbH, Postfach 3640, D-7500 Karlsruhe 1, Federal Republic of Germany

52. Bibliotheque, Centre de Recherches en Physique des Plasmas, Ecole Polytechnique Fédérale de Lausanne, 21 Avenue des Bains, CH-1007 Lausanne, Switzerland

53. R. Aymar, CEN/Cadarache, Departement de Recherches sur la Fusion Contrôlée, F-13108 Saint-Paul-lez-Durance Cedex, France

54. Bibliothèque, CEN/Cadarache, F-13108 Saint-Paul-lez-Durance Cedex, France

55. Library, JET Joint Undertaking, Abingdon, Oxfordshire OX14 3EA, England

56. Library, FOM-Instituut voor Plasmafysica, Rijnhuizen, Edisonbaan 14, 3439 MN Nieuwegein, The Netherlands

57. Library, National Institute for Fusion Science, Chikusa-ku, Nagoya 464-01, Japan

58. Library, International Centre for Theoretical Physics, P.O. Box 586, I-34100 Trieste, Italy

59. Library, Centro Richerche Energia Frascati, C.P. 65, I-00044 Frascati (Roma), Italy

60. Library, Plasma Physics Laboratory, Kyoto University, Gokasho, Uji, Kyoto 611, Japan

61. Plasma Research Laboratory, Australian National University, P.O. Box 4, Canberra, A.C.T. 2601, Australia

62. Library, Japan Atomic Energy Research Institute, Naka Fusion Research Establishment, 801-1 Mukoyama, Naka-machi, Naka-gun, Ibaraki-ken, Japan

Argonne National Laboratory, 9700 South Cass Avenue, Argonne, IL 60439

63. P. A. Finn, Bldg. 205

64. Y. Gohar, Bldg. 205

65. A. M. Hassanein, Bldg. 205

66. R. F. Mattas 
General Atomics, P.O. Box 85608, San Diego, CA 92138-5608

67. D. Overskei

68. F. A. Puhn

69. K. R. Schultz

70. R. D. Stambaugh

71. J. C. Wesley

Lawrence Livermore National Laboratory, P.O. Box 5511, Livermore, CA 94550

72. W. L. Barr, L-644

73. R. H. Bulmer, L-644

74. R. S. Devoto, L-644

75. J. N. Doggett, L-441

76. P. F. Dubois, L-630

77. M. E. Fenstermacher, L-644

78. J. Fink, L-644

79. A. Glass

80. C. D. Henning

81. S. K. Ho, L-644

82. J. A. Kerns, L-538

83. J. D. Lee, L-644

84. B. G. Logan, L-644

85. J. R. Miller, L-643

86. J. O. Myall, L-635

87. L. J. Perkins, L-644

Los Alamos National Laboratory, P.O. Box 1663, Los Alamos, NM 87545

88. C. B. Bathke, MS-F641

89. R. L. Miller, MS-F641

90. R. K. Linford

91. R. S. Willms, MS-C348

Massachusetts Institute of Technology, 77 Massachusetts Avenue, Cambridge, MA 02139

92. D. B. Montgomery, Plasma Fusion Center

93. M. Porkolab, Plasma Fusion Center

94. R. J. Thome, Francis Bitter National Magnet Laboratory

Ontario Hydro, 700 University Avenue, Toronto, Ontario M5G 1X6, Canada

95. A. Busigin, H11-F26

96. K. M. Kalyanam, H11-F26

Princeton Plasma Physics Laboratory, P.O. Box 451, Princeton, NJ 08544

97. F. W. Perkins

98. D. E. Post

99. P. H. Rutherford 
100. G. V. Sheffield

101. K. M. Young

102. F. Engelmann, NET Team, Max-Planck-Institut fr Plasmaphysik, D-8046 Garching, Federal Republic of Germany

103. N. Fugisawa, Japan Atomic Energy Research Institute, Naka Fusion Research Establishment, Naka-machi, Naka-gun, Ibaraki-ken 311-02, Japan

104. G. E. Gorker, 1727 Marlyn Way, San Jose, CA 95125

105. J. Holdren, University of California, Berkeley, CA 94720

106. T. Kammash, Department of Nuclear Engineering, University of Michigan, Cooley Building, North Campus, Ann Arbor, MI 48109

107 G. H. Miley, Nuclear Engineering Laboratory, University of Illinois, 103 South Goodwin Avenue, Urbana, IL 61801

108. R. R. Stasko, Canadian Fusion Fuels Technology Project, 2700 Lakeshore Road West, Mississauga, Ontario L5J 1K3, Canada

109. L. E. Strawbridge, Westinghouse Electric Corporation, P.O. Box 158, Madison, PA 15663

110. F. Thomas, B-20-5, Grumman Corporation, Bethpage, NY 11714

111. C. E. Wagner, JAYCOR, P.O. Box 85154, San Diego, CA 92138

112. H. Weitzner, Courant Institute of Mathematical Sciences, New York University, 251 Mercer Street, New York, NY 10012

113-161. Given distribution as shown in OSTI-4500, Magnetic Fusion Energy (Category Distribution UC-423 and UC-424, Magnetic Fusion Systems and Magnetic Reactor Materials) 

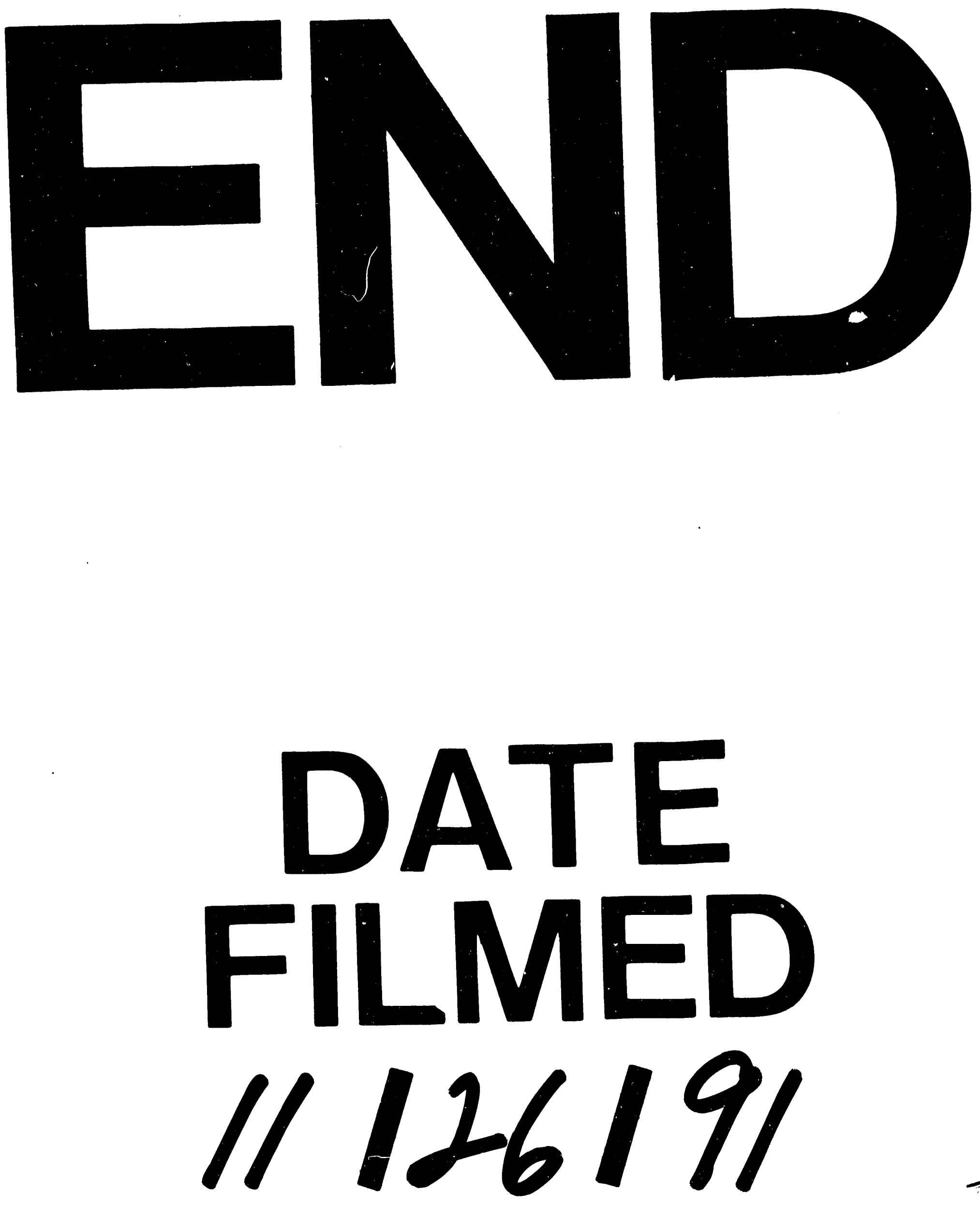

T" 
